\title{
GEOMETRY OF HOMOCLINIC CONNECTIONS IN A PLANAR CIRCULAR RESTRICTED THREE-BODY PROBLEM
}

\author{
MARIAN GIDEA AND JOSEP J. MASDEMONT
}

\begin{abstract}
The stable and unstable invariant manifolds associated with Lyapunov orbits about the libration point $L_{1}$ between the primaries in the planar circular restricted three-body problem with equal masses are considered. The behavior of the intersections of these invariant manifolds for values of the energy between the one of $L_{1}$ and that of the other collinear libration points $L_{2}$ and $L_{3}$ is studied using symbolic dynamics. Homoclinic orbits are classified according to the number of turns about the primaries.
\end{abstract}

\section{INTRODUCTION}

In this paper we study, mainly through numerical methods, the dynamics in the planar circular restricted three-body problem with equal masses. We describe a method to find invariant manifolds of Lyapunov orbits near the libration point $L_{1}$ (located between the primaries) and the behavior of the invariant manifolds and homoclinic trajectories for the energy levels between the ones of $L_{1}$ and $L_{2}$, where $L_{1}, L_{2}$ and $L_{3}$ refer to the collinear libration points. The orbits for these energy levels are forced to move in a neighborhood of the primaries. We obtain a classification of the homoclinic orbits with respect to the number of turns made by the body of infinitesimal mass around the primaries. At the same time, we provide numerical evidence for the existence of horseshoes with infinitely many branches, as well as symbolic dynamics over infinitely many symbols, corresponding to trajectories with prescribed itineraries.

The behavior of the invariant manifolds of Lyapunov orbits near $L_{1}$ in the planar restricted three-body problem - in the case when one primary has relative mass close to zero - was described through analytical methods in [7, 19, 8, 27], and through numerical methods in [16]. In this paper we show that the mechanisms and the qualitative behavior identified in the papers mentioned above survive to the case of the largest possible value of the relative mass. Techniques similar to those described in this paper were applied in [12] to find homoclinic and heteroclinic orbits between $L_{4}$ and $L_{5}$ for the planar restricted three-body problem, for varying values of the mass ratio.

The problem investigated here can serve as a benchmark for the design of space missions in which a spacecraft travels from one point to another on an optimal route, without lingering unnecessarily neither around the libration points nor around the small primary (see [5]). Other related applications can be found in [3, 22].

The model under consideration can be used in studying the dynamics of planets orbiting systems of binary stars (see $[14,11]$ ), in which case the primaries have

The work of J. J. M. has been supported by the Spanish grant BFM2003-09504 and the Catalan grant 2003XT-00021. 
masses of the same order. Here we mention that the first observed planet in a tight binary star system (Gamma Cephei) was found by A. Hatzes of Thueringer Landessternwarte Tautenburg and B. Cochran from the University of Texas, Austin and the McDonald Observatory, in October 2002.

Another possible application regards mass transfers in binary stars. Mass transfer commonly occurs in tight binary star systems. For example, mass transfer was observed optically in the binary star system Algol (Betta Persei). Algol is a short period binary well known for its transient accretion disk, in contrast to steady accretion disks associated to longer period systems. Ballistic and hydrodynamic models for mass transfers in Algol were studied in [18, 4]. We anticipate that the mechanisms described in this paper can also be used to analyze mass transfers phenomena of binary stars. Similar methods have been successfully employed in $[9,10]$ in the study of the transport of material throughout the solar system.

\section{The Planar Circular ReStricted three-Body PROBlem}

In the planar circular restricted three-body problem, two bodies of large masses $m_{1}$ and $m_{2}$ (called primaries) move along a circular orbit about their common center of mass, while a third body, of infinitesimal mass, moves in the plane of the circle, subject to the gravitational attraction of the primaries. The motion of the primaries is not affected by the motion of the infinitesimal body. One can assume that the angular velocity of the primaries is normalized to one. It is customary to study the motion of the infinitesimal body with respect to a reference frame that rotates with the primaries at the same angular velocity (called co-rotating, or synodical frame). See [26]. Relative to such a frame, the position of the primaries are $(-\mu, 0,0)$ and $(1-\mu, 0,0)$, where $\mu=m_{1} /\left(m_{1}+m_{2}\right)$ is the relative mass of the first primary. The motion of the infinitesimal body is described by

$$
\ddot{x}=2 \dot{y}+\frac{\partial V}{\partial x}, \quad \ddot{y}=-2 \dot{x}+\frac{\partial V}{\partial y},
$$

where the function $V$ (the effective potential) is given by

$$
V(x, y)=\frac{1}{2}\left(x^{2}+y^{2}\right)+\frac{1-\mu}{r_{1}}+\frac{\mu}{r_{2}} .
$$

Here $r_{1}=\left((x+\mu)^{2}+y^{2}\right)^{1 / 2}$ and $r_{2}=\left((x-1+\mu)^{2}+y^{2}\right)^{1 / 2}$ are the distances from the infinitesimal body to the primaries. The terms $2 \dot{y},-2 \dot{x}$ in the expressions of $\ddot{x}$ and $\ddot{y}$ in (2.1) represent the Coriolis force, while the term $(1 / 2)\left(x^{2}+y^{2}\right)$ in $(2.2)$ corresponds to the centrifugal force due to the rotation of the frame.

This motion has a first integral (called the Jacobi integral) given by

$$
C(x, y, \dot{x}, \dot{y})=-\left(\dot{x}^{2}+\dot{y}^{2}\right)+2 V(x, y) .
$$

The energy function is given by

$$
H(x, y, \dot{x}, \dot{y})=-\frac{1}{2} C(x, y, \dot{x}, \dot{y}) .
$$

The three-dimensional energy manifold

$$
\mathcal{M}_{h}=\{H(x, y, \dot{x}, \dot{y})=h\}, \text { with } h=\text { constant, }
$$

is non-compact (see Figure 1). The projection of an energy manifold onto the position space $(x, y)$ is called a Hill's region, and its boundary is a zero velocity curve. Any trajectory is confined to the Hill's region corresponding to the energy 


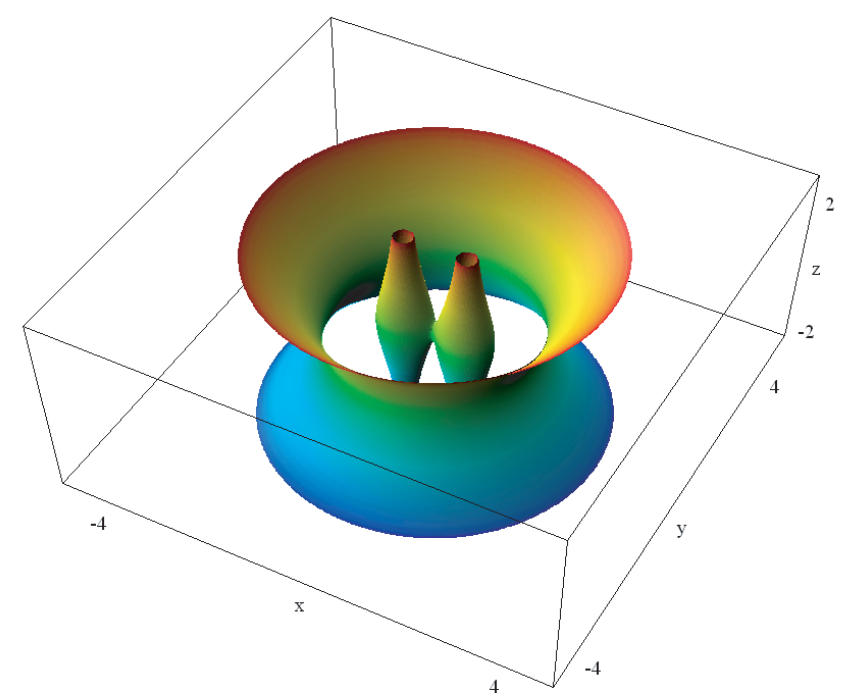

Figure 1. An $(x, y, \dot{x})$-plot of an energy manifold for $\mu=1 / 2$. To any point on this surface it corresponds a pair of points on the energy manifold, whose $\dot{y}$-coordinates result from the energy condition.

level of that trajectory. The shaded region in Figure 2 represents a Hill's region for an energy level typical of those considered below.

In the sequel, we restrict to the situation when the primaries have equal masses, that is, $\mu=1 / 2$.

Notice that the equations of motion are symmetric with respect to

$$
(x, y, \dot{x}, \dot{y}, t) \longrightarrow(x,-y,-\dot{x}, \dot{y},-t),
$$

and, since $\mu=1-\mu$, also with respect to

$$
(x, y, \dot{x}, \dot{y}, t) \longrightarrow(-x,-y,-\dot{x},-\dot{y}, t) .
$$

Any orbit either possesses these symmetries, or it has symmetric partners.

The equilibrium points of the differential equations (2.1) are given by the critical points of $V$. There are five equilibrium points for this problem: three of them, which we call $L_{1}, L_{2}$ and $L_{3}$, are collinear with the primaries (with $L_{1}$ located in between), while the other two, which we call $L_{4}$ and $L_{5}$, form equilateral triangles with the primaries. The distance from $L_{1}$ to the less massive primary is given by the only positive solution to Euler's quintic equation (see [26])

$$
\gamma^{5}-(3-\mu) \gamma^{4}+(3-2 \mu) \gamma^{3}-\mu \gamma^{2}+2 \mu \gamma-\mu=0,
$$

while the distance from $L_{2}$ to the less massive primary is given by the only positive solution of

$$
\gamma^{5}+(3-\mu) \gamma^{4}+(3-2 \mu) \gamma^{3}-\mu \gamma^{2}-2 \mu \gamma-\mu=0 .
$$

In our case, the solution to the first equation is the distance from $L_{1}$ to either primary, and the solution to the second equation is the distance from either $L_{2}$ 


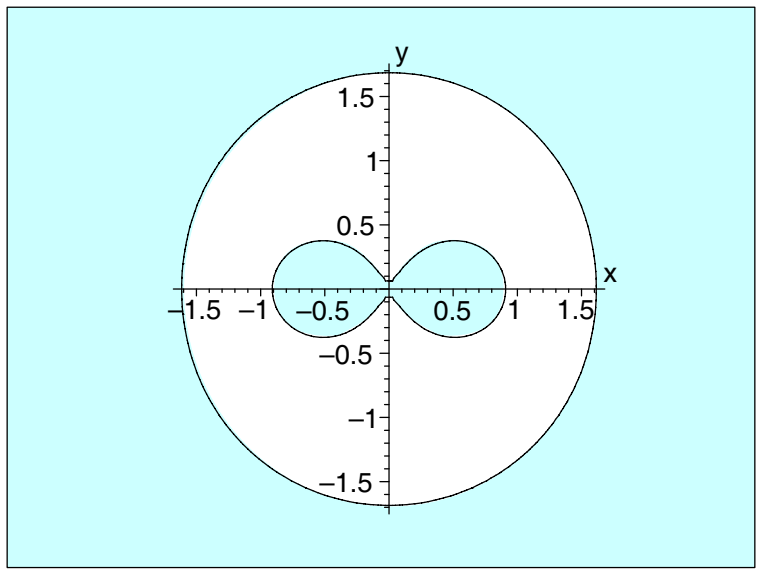

FiguRE 2. A Hill's region for $\mu=1 / 2$. The motion for the level of energy considered is restricted to the the shaded area.

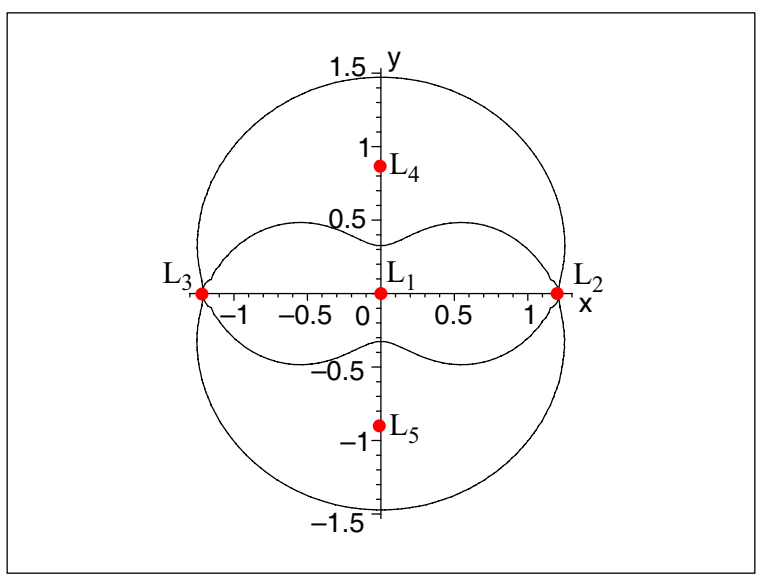

Figure 3. The five libration points for $\mu=1 / 2$ and the zero velocity curve for $C=C_{2}$.

and $L_{3}$ to the closest primary. We obtain $L_{1}=(0,0), L_{2}=(1.198406145,0)$, $L_{3}=(-1.198406145,0), L_{4}=(0,0.8660254038)$, and $L_{5}=(0,-0.8660254038)$.

The point $L_{1}$ corresponds to a Jacobi constant of $C_{1}=4, L_{2}$ and $L_{3}$ to $C_{2}=$ $C_{3}=3.456796224$, and $L_{4}$ and $L_{5}$ to $C_{4}=C_{5}=2.75$. Their position is shown in Figure 2. The figure also shows the zero velocity curve enclosing the Hill region corresponding to $C_{2}$. In order to have a Hill's region of the type as shown in Figure 2 (that is, with a bottle-neck channel through which the infinitesimal body could move from left to right and viceversa, but not to the outside part), the Jacobi constant should satisfy $C_{2}<C<C_{1}$. 


\section{THE DYNAMiCS NEAR $L_{1}$}

The equations (2.1) are equivalent to the following first order system

$$
\begin{aligned}
\dot{x} & =u, & \dot{y} & =v, \\
\dot{u} & =2 v+\frac{\partial V}{\partial x}, & \dot{v} & =-2 u+\frac{\partial V}{\partial y} .
\end{aligned}
$$

The linearization of this system at $L_{1}$ yields

$$
\begin{array}{ll}
\dot{x}=u, & \dot{y}=v, \\
\dot{u}=2 v+17 x, & \dot{v}=-2 u-7 y .
\end{array}
$$

The eigenvalues of this linear system are $\pm \lambda_{0}$ and $\pm i \nu_{0}$, where $\lambda_{0}=\sqrt{3+8 \sqrt{2}}=$ 3.783346205 and $\nu_{0}=|\sqrt{3-8 \sqrt{2}}|=2.883350220$. The corresponding eigenvectors are

$$
\begin{aligned}
v_{\lambda_{0}} & =\left(1,-\sigma, \lambda_{0},-\lambda_{0} \sigma\right), \quad v_{-\lambda_{0}}=\left(1, \sigma,-\lambda_{0},-\lambda_{0} \sigma\right), \\
v_{i \nu_{0}} & =\left(1, i \tau, i \nu_{0},-\nu_{0} \tau\right), \quad v_{-i \nu_{0}}=\left(1,-i \tau,-i \nu_{0},-\nu_{0} \tau\right),
\end{aligned}
$$

where $\sigma=0.3550152901, \tau=4.389634724$. Using these eigenvectors as a new basis, and new coordinates $\left(\xi^{1}, \xi^{2}, \eta^{1}, \eta^{2}\right)$ with respect to this basis, the equations $(3.2)$ become

$$
\dot{\xi}^{1}=\lambda_{0} \xi^{1}, \quad \dot{\xi}^{2}=-\lambda_{0} \xi^{2}, \quad \dot{\eta}^{1}=\nu_{0} \eta^{2}, \quad \dot{\eta}^{2}=-\nu_{0} \eta^{1} .
$$

The solutions to the above equations with initial conditions $\xi_{0}^{1}, \xi_{0}^{2}, \eta_{0}^{1}, \eta_{0}^{2}$ are

$$
\xi^{1}(t)=\xi_{0}^{1} e^{\lambda_{0} t}, \quad \xi^{2}(t)=\xi_{0}^{2} e^{-\lambda_{0} t}, \quad \eta^{1}(t)+i \eta^{2}(t)=\left(\eta_{0}^{1}+i \eta_{0}^{2}\right) e^{-i \nu_{0} t} .
$$

This shows that the dynamics near $L_{1}$ is of saddle-center type, with 2-dimensional center direction, 1-dimensional unstable direction, and 1-dimensional stable direction.

Going back to the $(x, y, \dot{x}, \dot{y})$-coordinate system, the periodic orbits of the linearized system are elliptical orbits of the form

$$
x(t)=\alpha \cos \left(\nu_{0} t+\phi_{0}\right), \quad y(t)=\kappa \alpha \sin \left(\nu_{0} t+\phi_{0}\right),
$$

where $\alpha$ is the amplitude of the orbit, $\nu_{0}$ is the frequency, $\phi_{0}$ is the phase, and $\kappa=\sqrt{1-e^{2}}$, where $e$ is the eccentricity of the orbit. By the Lyapunov Center Theorem (see [1]), there exist periodic orbits for the original system which, in the limit, have frequencies related to $\nu_{0}$. We use Lindstedt-Poincaré expansions to obtain the Lypunov orbits to the original equations that now are written in this form:

$$
\begin{aligned}
\ddot{x}-2 \dot{y}-17 x & =\frac{\partial}{\partial x} \sum_{n \geq 3} c_{n} \rho^{n} P_{n}\left(\frac{x}{\rho}\right), \\
\ddot{y}+2 \dot{x}+7 y & =\frac{\partial}{\partial y} \sum_{n \geq 3} c_{n} \rho^{n} P_{n}\left(\frac{x}{\rho}\right),
\end{aligned}
$$

where $\rho=\sqrt{x^{2}+y^{2}}, P_{n}(t)=\frac{1}{2^{n} n !} \frac{d^{n}}{d t^{n}}\left[\left(t^{2}-1\right)^{n}\right]$ is the Legendre polynomial of degree $n$, and $c_{n}=4\left(1+(-1)^{n}\right)$, for $n \geq 2$. The coefficients $c_{n}$ in the case $\mu=1 / 2$, follow from a general formula described in [23]. The equations (3.4) are obtained from (2.1) by scaling the distance by $1 / 2$ (the distance from $L_{1}$ to any primary is taken as a new unit of distance) and then expanding the resulting equations using the Legendre polynomials. The transformation can be found in $[15,20]$. 
In order to find periodic orbits near $L_{1}$, we look for formal series in powers of the amplitude $\alpha$ of the type

$$
\begin{aligned}
& x(t)=\sum_{i=1}^{\infty}\left[\sum_{|h| \leq i} x_{i h} \cos \left(h\left(\nu t+\phi_{0}\right)\right)\right] \alpha^{i}, \\
& y(t)=\sum_{i=1}^{\infty}\left[\sum_{|h| \leq i} y_{i h} \sin \left(h\left(\nu t+\phi_{0}\right)\right)\right] \alpha^{i} .
\end{aligned}
$$

The frequency $\nu$ does not have to be equal to the corresponding frequency $\nu_{0}$ of the periodic solution of the linearized system. Instead, $\nu$ can also be represented as a formal series in powers of $\alpha$ of the type

$$
\nu=\nu_{0}+\sum_{i=1}^{\infty} \nu_{i} \alpha^{i}
$$

In order to find periodic orbits, one has to solve recurrently for the coefficients $x_{i h}$, $y_{i h}$, and $\nu_{i}$ up to some reasonably large order. If these coefficients are computed up to a certain order $n-1$, then the expression of $x$ and $y$ from (3.5) truncated at $i=n-1$ are substituted in the right hand side of (3.4), producing terms of order $n$ in $\alpha$. Then the coefficients of order $n$ are computed by identifying the unknown coefficients from the left hand side with combinations of the already known coefficients from the right hand side. For details, see [15] and the references listed there. In this way, one can explicitly find Lyapunov orbits for energy levels sufficiently close to that of $L_{1}$. In the sequel, we will consider an energy level slightly above that of $L_{1}$.

\section{Surface OF SECTION}

We will consider the Poincaré first return map to the surface of section

$$
\Pi^{+}=\{(x, y, \dot{x}, \dot{y}) \mid x>1 / 2, \quad y=0, \quad \dot{y}>0\},
$$

where the $x$-coordinate is restricted to the interval between the right-hand side primary and $L_{2}$.

Any trajectory that intersects transversally the surface of section $\Pi^{+}$is uniquely determined, up to its sense, by the $(x, \dot{x})$-coordinates of the intersection point, since an initial condition for that trajectory can be obtained by letting $y=0$ and solving for $\dot{y}$ from the energy condition (2.4).

The condition for a trajectory to meet $\Pi^{+}$transversally is that the vector field associated with (3.1) is not perpendicular to the normal $(0,1,0,0)$ to $\Pi^{+}$, which translates into $\dot{y} \neq 0$. For a trajectory tangential to $\Pi^{+}$, the $(x, \dot{x})$-coordinates at tangency points should satisfy

$$
\begin{aligned}
(\dot{x})^{2} & =2 V(x, 0)-c \\
& =x^{2}+2 \frac{1-\mu}{x+\mu}+2 \frac{\mu}{x+\mu-1}-c,
\end{aligned}
$$

where $c$ is the corresponding Jacobi constant. The tangency curves depend on both the energy level and the surface of section considered. This equation represents a curve, which we will refer to as the tangency curve (see Figure 4). 


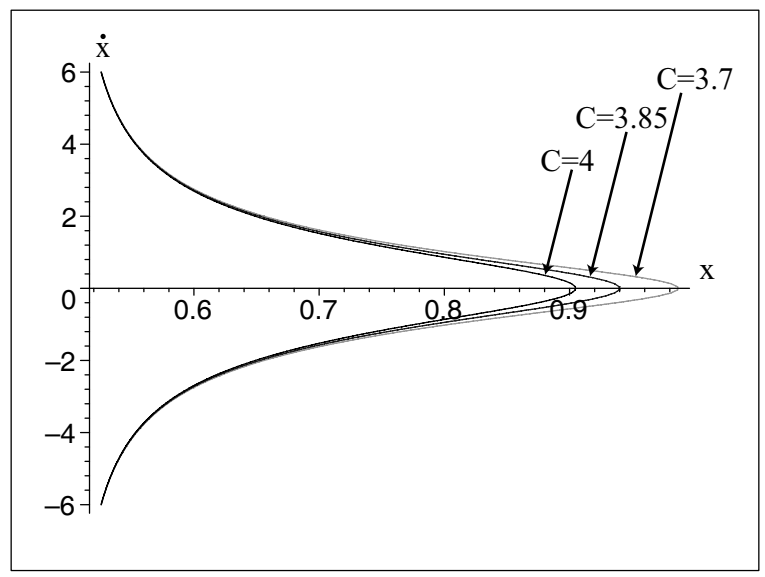

Figure 4. Tangency curves in the surface of section $\Pi^{+}$, for Jacobi constants $C=4, C=3.85, C=3.7$.

The equations (2.1) along the tangency curve in $\Pi^{+}$are

$$
\begin{aligned}
& \ddot{x}=\quad x-\frac{(1-\mu)(x+\mu)}{(x+\mu)^{3}}-\frac{\mu(x+1-\mu)}{(x+1-\mu)^{3}}, \\
& \ddot{y}=-2 \dot{x} .
\end{aligned}
$$

There is only one intersection of the tangency curve with the $x$-axis in $\Pi^{+}$, given by the only root between the right-hand side primary and $L_{2}$ of the quartic equation

$$
\left(x^{2}-c\right)(x+\mu)(x+1-\mu)+2 x+2(-1+2 \mu)=0 \text {. }
$$

At this point $\dot{x}=0$ hence $\ddot{y}=0$. Note that $\ddot{y} \neq 0$ at any other point of the tangency curve.

Assume by contradiction that $\dddot{y}=0$ at the intersection point of the tangency curve with the $x$-axis. Since

$$
\frac{\partial V}{\partial y}=y\left[2-\frac{1-\mu}{\left.\left((x+\mu)^{2}+y^{2}\right)\right)^{3 / 2}}-\frac{\mu}{\left.\left((x-1+\mu)^{2}+y^{2}\right)\right)^{3 / 2}}\right] \text {, }
$$

$\frac{\partial V}{\partial y}=0$ and $\frac{d}{d t} \frac{\partial V}{\partial y}$ at any tangency point. Since $\ddot{y}=-2 \dot{x}+\frac{\partial V}{\partial y}$,

$$
\dddot{y}=-2 \ddot{x}+\frac{d}{d t} \frac{\partial V}{\partial y},
$$

so $\dddot{y}=0$ implies $\ddot{x}=0$. Using $\ddot{x}=2 \dot{y}+\partial V / \partial x$, we infer that $\partial V / \partial x=0$ at the tangency point. This means the tangency point is a critical point of $V$, i.e., a libration point, which is impossible. Thus the third order derivative of $y$ cannot be equal to zero at a tangency point.

We have obtained the following classification of trajectories in terms of their type of intersection with the surface of section:

- Each trajectory that meets the surface of section at points off the tangency curve is transverse to the surface of section.

- Each trajectory that meets the surface of section at points along the tangency curve with $\dot{x} \neq 0$ exhibits a quadratic tangency with the surface of section. 


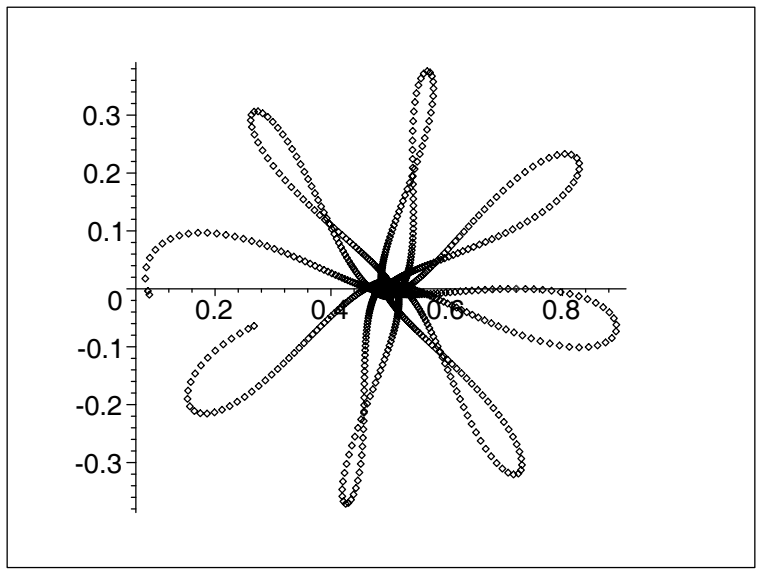

FiguRE 5 . Trajectory tangent to $\Pi^{+}$, projected onto the $x y$-plane.

- There are precisely two trajectories that meet the surface of section at the point on the tangency curve with $\dot{x}=0$, and these two trajectories exhibit cubic tangencies with the surface of section.

A trajectory exhibiting a quadratic tangency is shown in Figure 5.

We denote by $\Phi^{+}: \Pi^{+} \rightarrow \Pi^{+}$the Poincaré first return map to $\Pi^{+}$: for $x \in \Pi^{+}$, $\Phi^{+}(x)$ represents the first point of intersection of the forward trajectory of $x$ with $\Pi^{+}$. This map is only partially defined, since it is possible for a point $x \in \Pi^{+}$that its forward trajectory makes a transfer to the region $x<0$ and never returns to $\Pi^{+}$. It is also possible for a point $x \in \Pi^{+}$that its forward trajectory makes a transfer to the region $x<0$ and and returns to $\Pi^{+}$after a while; in this case $\Phi^{+}(x)$ is well defined, however it provides no information on the behavior of the trajectory of $x$ in $x<0$. In this situation, it is convenient to consider the surface of section $\Pi^{-}$, symmetric to $\Pi^{+}$about $L_{1}$, given by

$$
\Pi^{-}=\{(x, y, \dot{x}, \dot{y}) \mid x<-1 / 2, \quad y=0, \quad \dot{y}<0\},
$$

where the $x$-coordinate is restricted to the interval between the left-hand side primary and $L_{3}$. In a similar fashion, we consider the partially defined map $\Phi^{-}: \Pi^{-} \rightarrow \Pi^{-}$, the Poincaré first return map to $\Pi^{-}$. Since $\Phi^{-}$presents the same inconvenience as $\Phi^{+}$, we also define

$$
\Phi: \Pi^{+} \cup \Pi^{-} \rightarrow \Pi^{+} \cup \Pi^{-},
$$

where for $x \in \Pi^{+} \cup \Pi^{-}, \Phi(x)$ represents the first point of intersection of the forward trajectory of $x$ with either $\Pi^{-}$or $\Pi^{+}$. Except for the case when the trajectory of $x$ collides with one of the primaries (see Section 8 ), $\Phi(x)$ is well defined for all $x$. If $x \in \Pi^{+}$and $\Phi(x) \in \Pi^{+}$, then $\Phi(x)=\Phi^{+}(x)$; if $x \in \Pi^{-}$and $\Phi(x) \in \Pi^{-}$, then $\Phi(x)=\Phi^{-}(x)$. We can take iterates of the map $\Phi$, in which case $\Phi^{N}(x)$ represents the $N$-th intersection of the forward trajectory of $x$ with $\Pi^{+} \cup \Pi^{-}$, after performing a total number of $N$ revolutions about either primary. 


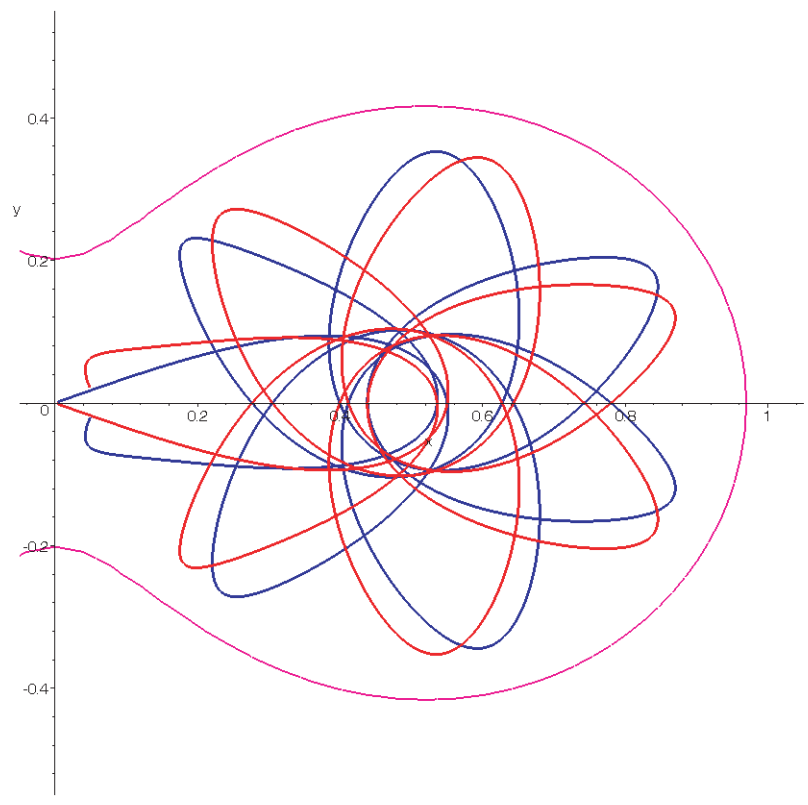

FiguRE 6. The stable and unstable manifolds of $L_{1}$, projected onto the $x y$-plane.

\section{Stable And unstable manifolds of Lyapunov orbits}

For the level of energies considered, the Lyapunov orbits have 2-dimensional stable and unstable manifolds. If we let the energy level tend to that of $L_{1}$, the stable and unstable manifolds reduce to 1-dimensional stable and unstable manifolds $W^{s}\left(L_{1}\right)$ and $W^{u}\left(L_{1}\right)$ of the libration point $L_{1}$, respectively. Note that the energy manifold is degenerate at $L_{1}$, so $L_{1}$ is not a hyperbolic fixed point. Nevertheless $W^{u}\left(L_{1}\right)$ and $W^{s}\left(L_{1}\right)$ are tangent to $v_{\lambda_{0}}$ and $v_{-\lambda_{0}}$ at $L_{1}$, respectively. Numerical integration in the stable and the unstable directions seem to indicate that $W^{u}\left(L_{1}\right)$ and $W^{s}\left(L_{1}\right)$ do not to intersect (hence they do not coincide), but they get arbitrarily close to one another. They appear to intersect the surface of section only transversally, at points off the tangency curve. It also appears $W^{u}\left(L_{1}\right)$ and $W^{s}\left(L_{1}\right)$ fill up densely the same positive measure region of the energy manifold. See Figure 6 and Figure 7. This would constitute an obstruction against integrability: it would imply that there is no other real analytic integral besides the Jacobi integral. However, it seems quite difficult to verify, either analytically or numerically, that the stable and unstable manifolds of $L_{1}$ do not coincide yet get arbitrarily close one to the other.

The region defined by the intersections of the stable and unstable manifolds with the surface of section appears to be stochastic. The role of this region in the dynamics will be discussed below. We note that this region is similar to the 'maple leaf' stochastic region described for Hill's problem in [24].

Now we consider the stable and unstable manifolds of Lyapunov orbits. Each Lyapunov orbit $T_{C}$ (which is a 1-dimensional object), corresponding to a Jacobi constant $C$, has a 2-dimensional stable manifold $W^{s}\left(T_{C}\right)$, and a 2-dimensional unstable manifold $W^{u}\left(T_{C}\right)$. The manifolds $W^{s}\left(T_{C}\right)$ and $W^{u}\left(T_{C}\right)$ are contained in 


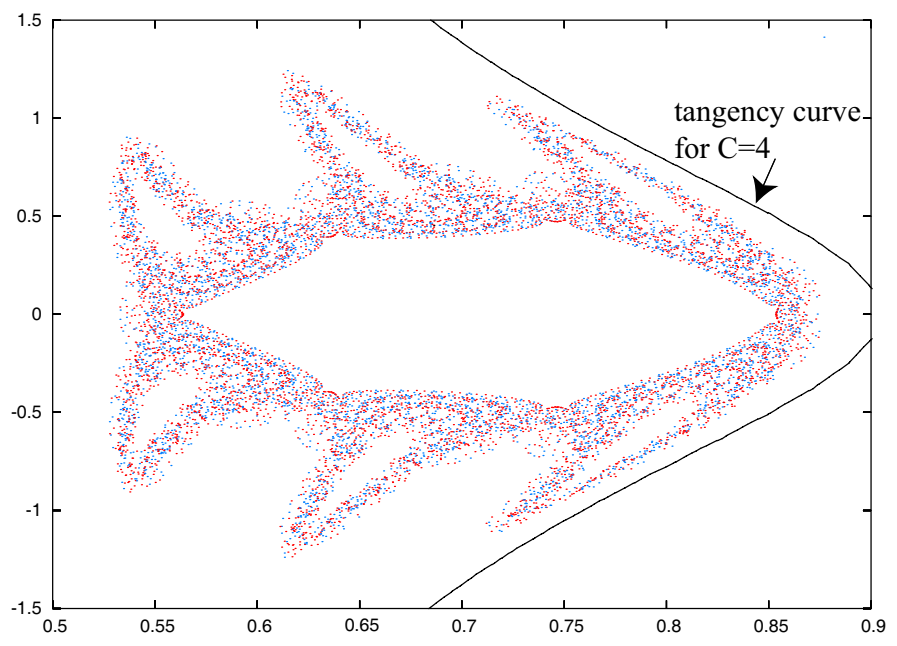

Figure 7 . The $(x, \dot{x})$-plot of the stable manifold of $L_{1}$ (and also of the unstable manifold of $L_{1}$ ) when it intersects the surface of section, and the tangency curve for $C=4$.

the same energy manifold as $T_{C}$ itself. The branches of each manifold are semicylinders symmetric with respect to $L_{1}$ (due to the symmetry (2.7)), and they consist of orbits asymptotic (in positive or negative time) to the Lyapunov orbit. Each point of the Lyapunov orbit can be uniquely described by a phase in $[0,2 \pi]$ (see Section 3). Therefore, we can parametrize the section plots using the initial phase. See Figure 9. The whole manifold, regarded as a tube of orbits, is computed using Lindstedt-Poincaré procedures, in a manner similar to that for the Lyapunov orbits. These orbits are solutions of the system given by (2.1), with initial conditions given by a point on the Lyapunov orbit and the corresponding stable or unstable direction at that point. These solutions are obtained by means of formal series in powers of amplitudes $\alpha_{1}, \alpha_{2}$ and $\alpha_{3}$, of the form:

$$
\begin{aligned}
& x(t)=\sum e^{(i-j) \lambda t}\left[x_{i j k}^{h} \cos \left(h\left(\nu t+\phi_{0}\right)\right)+\bar{x}_{i j k}^{h} \sin \left(h\left(\nu t+\phi_{0}\right)\right)\right] \alpha_{1}^{i} \alpha_{2}^{j} \alpha_{3}^{k}, \\
& y(t)=\sum e^{(i-j) \lambda t}\left[y_{i j k}^{h} \cos \left(h\left(\nu t+\phi_{0}\right)\right)+\bar{y}_{i j k}^{h} \sin \left(h\left(\nu t+\phi_{0}\right)\right)\right] \alpha_{1}^{i} \alpha_{2}^{j} \alpha_{3}^{k},
\end{aligned}
$$

where

$$
\nu=\sum \nu_{i j k} \alpha_{1}^{i} \alpha_{2}^{j} \alpha_{3}^{k}, \quad \lambda=\sum \lambda_{i j k} \alpha_{1}^{i} \alpha_{2}^{j} \alpha_{3}^{k} .
$$

Summation is extended over all $i, j, k$ and $h \in \mathbb{N}$. The amplitude $\alpha_{1}$ is the amplitude $\alpha$ in (3.5) associated with the Lyapunov orbit. The amplitudes $\alpha_{2}$ and $\alpha_{3}$ are associated with the hyperbolic manifolds. The coefficients $x_{i j k}^{h}, \bar{x}_{i j k}^{h}, y_{i j k}^{h}, \bar{y}_{i j k}^{h}, \nu_{i j k}, \lambda_{i j k}$ are computed recursively; many of them are actually zero due to symmetries. In our experiments, the series were truncated at the order 15.

When the unstable manifold crosses the section the first few times, the intersections are diffeomorphic copies of a circle. See Figure 8. Sometime after an intersection of the stable and unstable manifolds occurs (as we will describe in Section 6) the circles are destroyed. It appears that the cuts made by the stable and unstable manifolds are 'attached' to the stochastic region described above, which 


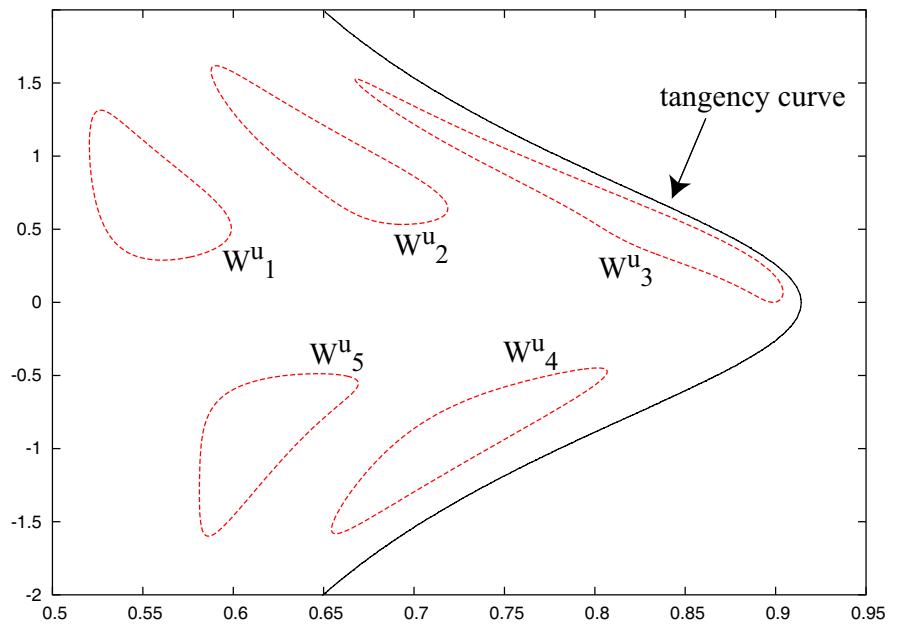

FIgURE 8. Successive cuts of the unstable manifolds and the tangency curve for the corresponding energy level.

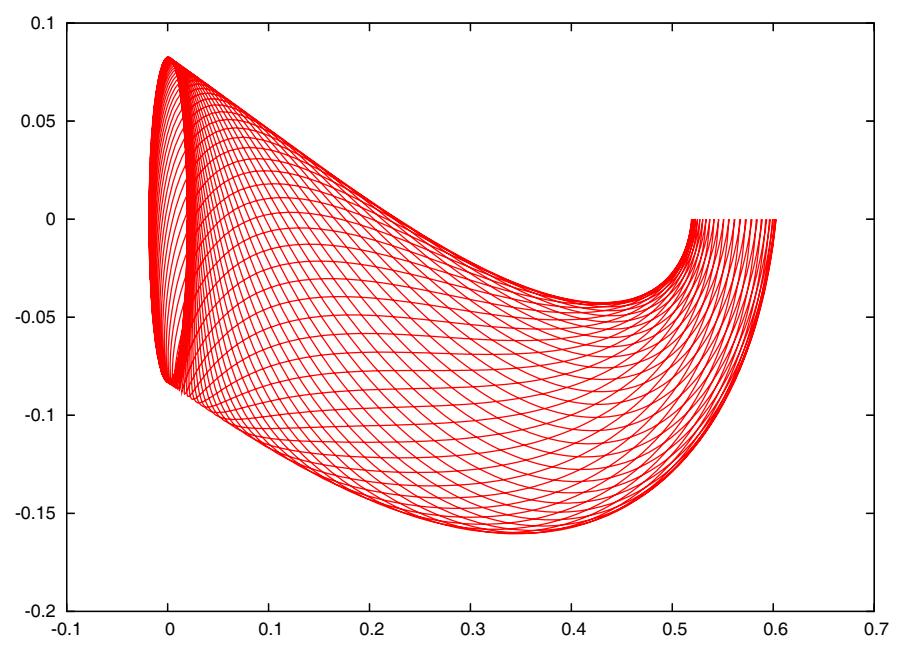

FiguRE 9. A branch of the unstable manifold of a Lyapunov orbit.

plays the role of a 'skeleton' for the regular behavior. See Figure 10. A similar behavior was described for Hill's problem in [24].

The stable and unstable manifolds of Lyapunov orbits are separatrices of the phase space (see $[6,16])$. They divide the phase space into two disjoint open regions. The trajectories inside the tube correspond to transit orbits, i.e., orbits that go from $x>0$ to $x<0$ and viceversa. The trajectories outside the tube bounce back to their region of origin to make at least one full turn around the primary. Consider, for example, the case when the stable and unstable manifolds of a Lyapunov orbit cross the surface of section $\Pi^{+}$into two intersecting circles. Then the surface of section is subdivided into four regions with behavior as illustrated in Figure 11. 


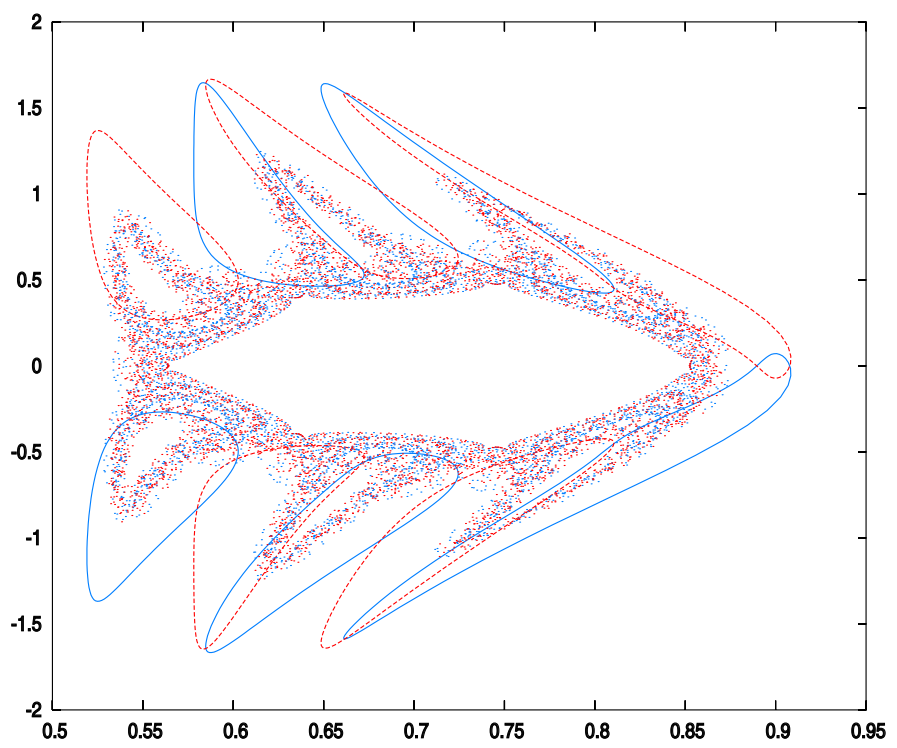

Figure 10. Successive cuts of the stable manifolds and unstable manifolds, and the stochastic region (the unstable manifold dashed red line, the stable manifold — solid blue line).

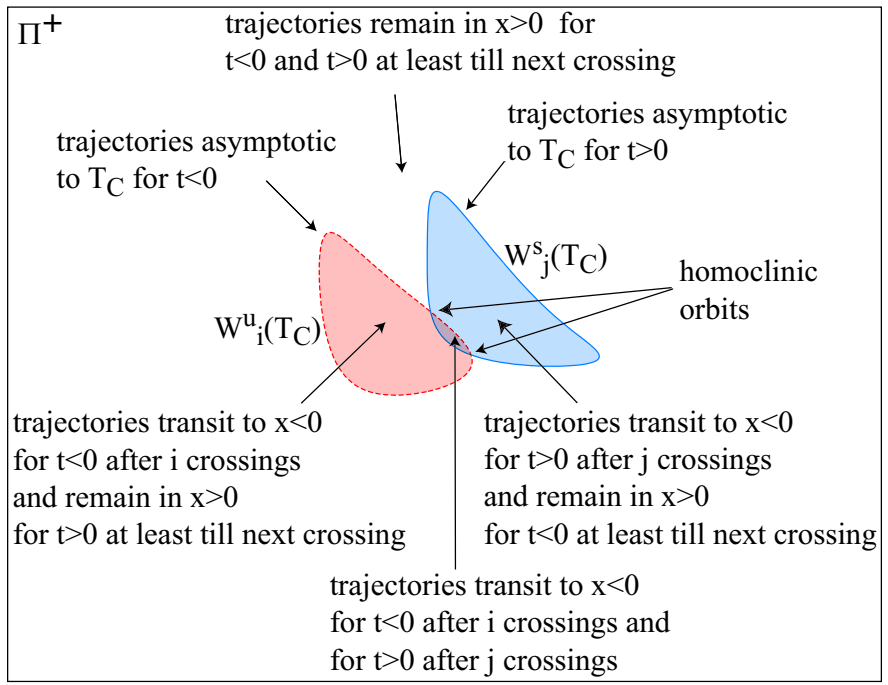

FiguRE 11. Transit and non-transit trajectories. Here $W_{i}^{u}\left(T_{C}\right)$ denotes the $i$-th crossing in forward time of $W^{u}\left(T_{C}\right)$ with $\Pi^{+}$, and $W_{j}^{s}\left(T_{C}\right)$ denotes the $j$-th crossing in backwards time of $W^{s}\left(T_{C}\right)$ with $\Pi^{+}$.

\section{TRANSVERSE HOMOCLINIC CONNECTIONS TO LYAPUNOV ORBITS}

An intersection of the stable and unstable manifolds could be transverse, which occurs generically, or tangential. The relative position of the circles determined by 
the intersections of $W^{u}\left(T_{C}\right)$ and $W^{s}\left(T_{C}\right)$ with $\Pi^{+}$determines the nature of the intersection between $W^{u}\left(T_{C}\right)$ and $W^{s}\left(T_{C}\right)$ in $\mathcal{M}_{h}$. The tangency of the circles implies the tangency between $W^{u}\left(T_{C}\right)$ and $W^{s}\left(T_{C}\right)$ at that point. To see this, note that the common tangent to the circles in the surface of section and the tangent to the homoclinic orbit (through the point of intersection of the two circles) are two linearly independent directions that span the tangent spaces of both manifolds. These manifolds are 2-dimensional, the energy manifold is 3-dimensional. The tangent spaces do not sum up to a 3-dimensional vector space, thus $W^{u}\left(T_{C}\right)$ and $W^{s}\left(T_{C}\right)$ cannot be transverse. Conversely, if the circles determined by the intersections of $W^{u}\left(T_{C}\right)$ and $W^{s}\left(T_{C}\right)$ with the $\Pi^{+}$intersect transversally at some point, so do $W^{u}\left(T_{C}\right)$ and $W^{s}\left(T_{C}\right)$ in $\mathcal{M}_{h}$.

An example of a transverse intersection of stable and unstable manifolds of a Lyapunov orbit is shown in Figure 12 (Jacobi constant $C=3.95$ ). Solid lines in the plot correspond to the stable manifold and dashed lines to the unstable one. For example, $W_{1}^{u}$ denotes the first intersection of the unstable manifold of the Lyapunov orbit with the surface section, and $W_{3}^{s}$ refers to the third intersection of the stable manifold when we integrate backwards. The intersection points are labeled with $A, B, \ldots$ (and also with $A^{\prime}, B^{\prime}, \ldots$, which correspond to homoclinic orbits symmetrical to those through $A, B, \ldots$ ). For example, $A, B$ represent the intersections of $W_{1}^{u}$ with $W_{5}^{s}$, that is, two homoclinic orbits that cross five times the surface of section. The other ones are:

\begin{tabular}{|c|c|c|c|}
\hline Points & $W_{*}^{u}$ & $W_{*}^{s}$ & crossings with the surface of section \\
\hline A, B & 1 & 5 & 5 \\
C, D & 2 & 5 & 6 \\
E, F & 2 & 4 & 5 \\
G, H & 3 & 4 & 6 \\
I, J & 3 & 3 & 5 \\
\hline
\end{tabular}

The homoclinic orbits $I$ and $J$ are symmetrical because they satisfy $\dot{x}=0$. For this level of energy, we conclude that 5 is the minimum number of crossings that a homoclinic orbit can have with the section. The orbit corresponding to $J$ is shown in Figure 13. It is clear that the set of points $A, B, E, F, I, J$ corresponds to only two different trajectories. Similarly, $C, D, G, H$ correspond to two homoclinic orbits having 6 crossings with the section.

We now discuss what happens to the intersections of $W_{*}^{u}$ and $W_{*}^{s}$ as the number of crossings of $W^{u}\left(T_{C}\right)$ with $\Pi^{+}$increases (and the number of crossings of $W^{s}\left(T_{C}\right)$ with $\Pi^{+}$in negative time decreases to keep the number of intersections of the homoclinic orbit the same). In positive time, when the unstable manifold turns around the right hand-side primary, the intersections up to the 5 -th encounter are diffeomorphic copies of a circle. The different trajectories that constitute the unstable tube travel at different speeds, so they will intersect the surface of section at different times, and the shape of the successive intersection will be progressively distorted. After the intersection of $W_{5}^{u}$ with $W_{1}^{s}$, the trajectories near the homoclinic orbit will be redirected back to the Lyapunov orbit, while the rest of the trajectories will keep turning around the mass. See Figure 14. According to the diagram shown in Figure 11, some of the redirected trajectories will go to the region $x<0$ and approach the branch of unstable manifold in that region, and some others will approach the branch of the unstable manifold in $x>0$. 


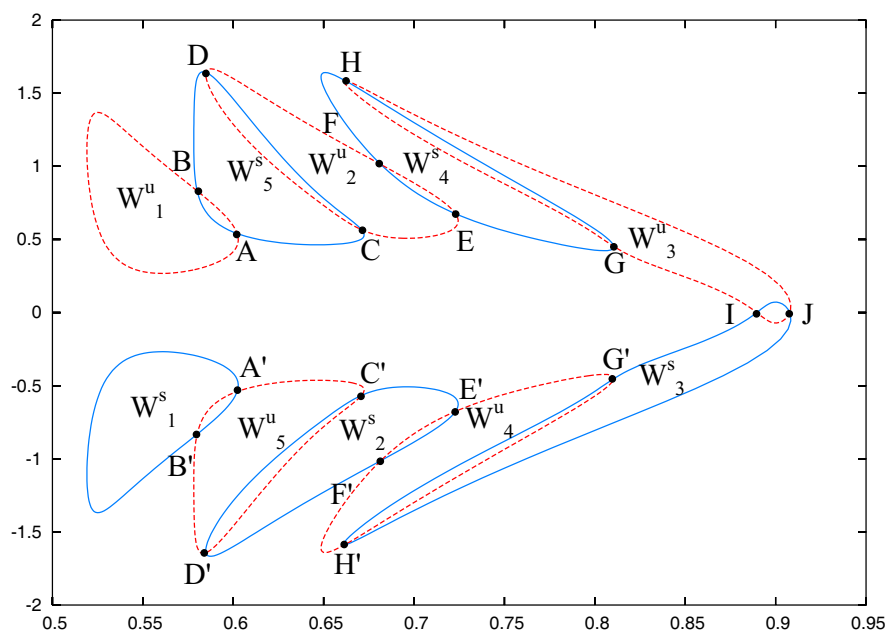

FiguRE 12. First transverse intersections of $W^{u}\left(T_{C}\right)$ and $W^{s}\left(T_{C}\right)$ within the $(x, \dot{x})$-plane, for Jacobi constant $C=3.95$.

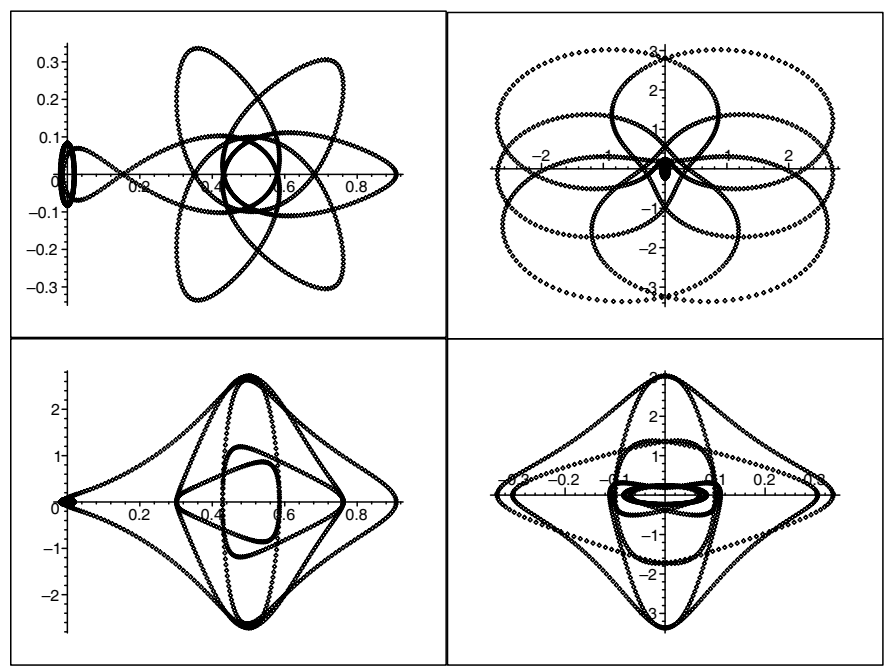

Figure 13. Symmetric homoclinic orbit for $C=3.95$ : $(x, y)$ view (upper left), $(\dot{x}, \dot{y})$-view (upper right), $(x, \dot{x})$-view (lower left), $(y, \dot{y})$-view (lower right).

We plot the time to reach the 6-th cut versus the initial phase for points on the Lyapunov orbit. There is a gap in this graph, between the two vertical asymptotes that mark the phases which give the homoclinic orbits. The phases inside the gap correspond to transit orbits. See Figure 15.

The successive intersections of the unstable tube with the surface of section after the 5-th crossing are no longer homeomorphic to a circle. Excluding a small neighborhood about the homoclinic orbit, the 6 -th crossing of the unstable manifold will consist of a segment of a circle, which endpoints are marked by $S$ and $T$ in 


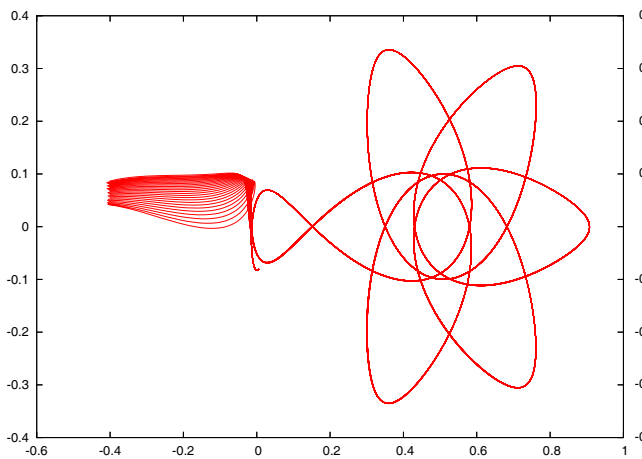

(a)

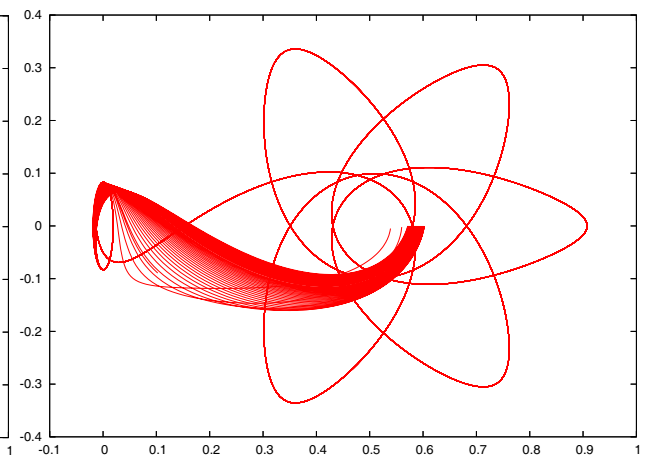

(b)

FiguRE 14. (a) Trajectories close to the homoclinic orbit transiting to $x<0$ and approaching the branch of the unstable manifold in $x<0$. (b) Trajectories close to the homoclinic orbit bouncing back to $x>0$ and approaching the branch of the unstable manifold in $x>0$.

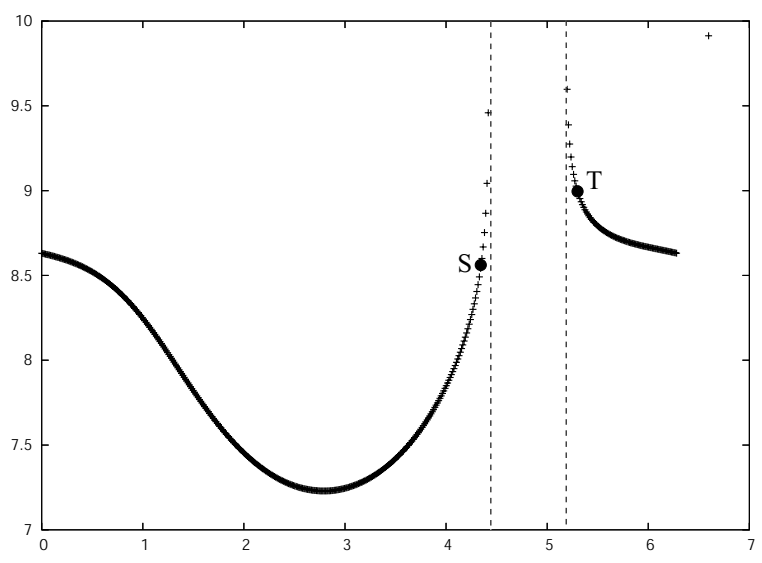

Figure 15. A graph of return time versus phase for points on the surface of section near the $W^{u}\left(T_{C}\right)$-crossing. The two asymptotes mark the phases which give the homoclinic orbits. The phases inside the gap correspond to orbits which transit to $x<0$.

Figure 16 (top), corresponding to the phases also marked by $S$ and $T$ in Figure 15. Within that neighborhood, there will be trajectories that make some number of turns near the Lyapunov orbit and then return to mimic the behavior of the unstable manifold prior to the 5-th crossing. The portion of $W_{6}^{u}$ corresponding to phases between $S$ and the first vertical asymptote and portion corresponding to phases between the second vertical asymptote and $T$ in Figure 15 represent two semi-open curves that wind around $W_{1}^{u}$ infinitely many times and approach $W_{1}^{u}$ asymptotically from the outside (due to the Lambda Lemma and to the fact that the unstable manifold does not self intersect). See Figure 16. Thus, letting aside the orbits of $W^{u}\left(T_{C}\right)$ which transit to $x<0$ after the 5 -th cut, $W_{6}^{u}$ consists of 


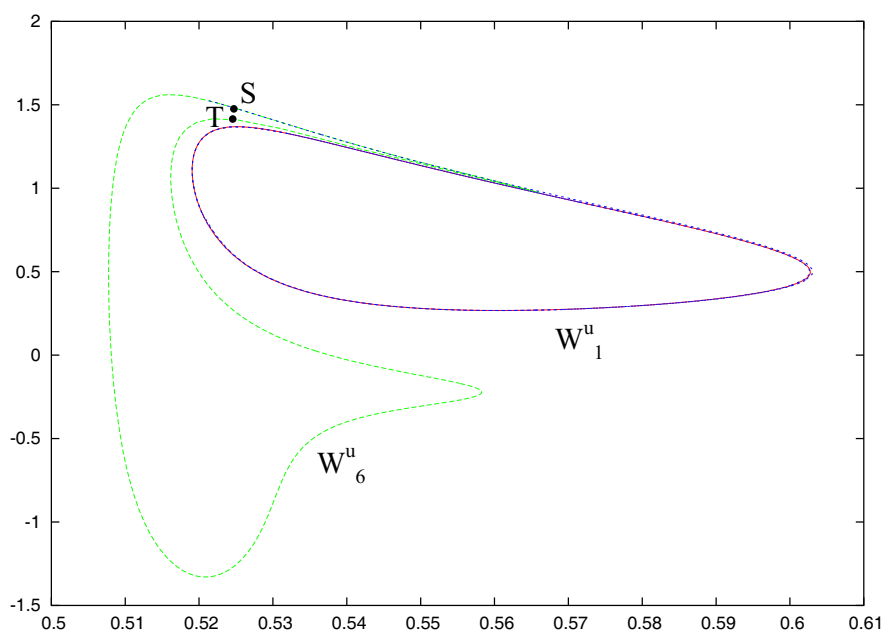

Figure 16. The curve between $S$ and $T$ represents the portion of $W_{6}^{u}$ with steady behavior. The remaining part consists of two open-ended curves that wind around $W_{1}^{u}$ infinitely many times. However the convergence is so fast that after a short time the curves are indistinguishable in the plot.

an open curve that winds infinitely many times towards $W_{1}^{u}$ in both ends, and so creates infinitely many approximative copies of $W_{1}^{u}$. The intersections of these copies with $W_{5}^{s}$ correspond to homoclinic orbits. Therefore, there are infinitely many such homoclinic orbits, each of them crossing $\Pi^{+}$exactly 10 times. That is, for this energy level, 10 is the smallest positive integer for which there exist infinitely many homoclinic orbits with a given number of crossings.

The orbits of $W^{u}\left(T_{C}\right)$ corresponding to the phases between the two asymptotes shown in Figure 15 form an open curve that transits to $x<0$ in forward time and follows closely the branch of the unstable manifold in $x<0$. This open curve approaches asymptotically the $(x<0)$-branch of the unstable manifold from the inside, with booth ends winding infinitely many times towards that branch. Then an intersection with the corresponding branch of the stable manifold occurs in an almost symmetrical way as in the case $x>0$. The open curve inside the $(x<0)$ branch of the unstable manifold is going to be cut by the $(x<0)$-branch of the stable manifold into infinitely many open curve segments. These curve segments represent orbits of $W^{u}\left(T_{C}\right)$ that transit most rapidly to $x<0$ and transit back most rapidly to $x>0$. In the Poincaré section $\Pi^{+}$, they will appear as an infinite collection of open curves winding around $W_{1}^{u}$ and approaching $W_{1}^{u}$ from the inside.

In the sequel, we present numerical evidence that the stable and unstable manifolds of any Lyapunov orbit always intersect. In the case of 'big' Lyapunov orbits (of Jacobi constant not very close to $C_{1}$ ), we generate numerically the stable and unstable manifolds of the Lyapunov orbits, and plot their intersection points within the surface of section. In the case of 'small' Lyapunov orbits (of Jacobi constant close to $C_{1}$ ), we argue the existence of intersection of the manifolds based on the 


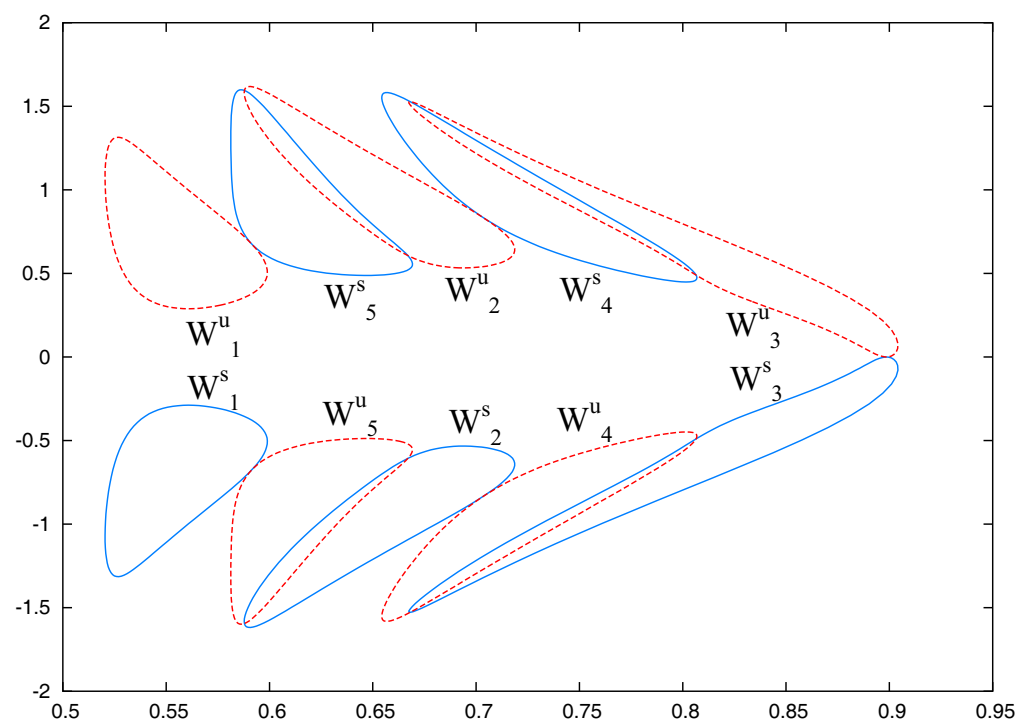

FiguRE 17. Tangential intersection of $W^{u}\left(T_{C}\right)$ and $W^{s}\left(T_{C}\right)$ along a homoclinic orbit with 5 crossings, for Jacobi constant $C=3.9556106472755$. There are some transverse intersection of $W^{u}\left(T_{C}\right)$ with $W^{s}\left(T_{C}\right)$, but they occur along two homoclinic trajectories with 6 crossings.

assumption that the stable and unstable manifolds of $L_{1}$ are disjoint and pass arbitrarily close to one another.

If we consider Lyapunov orbits for Jacobi constants $C$ tending to that for $L_{1}$, the number $N_{C}$ of crossings that a homoclinic orbit can have with the section increases with $C$. For example, when $C=3.95$, we have $N_{C}=5$. When $C$ increases, the pairs of $W_{*}^{u}\left(T_{C}\right)$ and $W_{*}^{s}\left(T_{C}\right)$ intersecting along the two homoclinic trajectories with 5 crossings are gradually pulled apart. For some bifurcation value of $C$, the two homoclinic orbits with 5 crossings coalesce into one, and the corresponding $W_{*}^{u}\left(T_{C}\right)$ and $W_{*}^{s}\left(T_{C}\right)$ intersect only tangentially. A tangential intersection of $W_{*}^{u}\left(T_{C}\right)$ and $W_{*}^{s}\left(T_{C}\right)$ is shown in Figure 17, for $C=3.9556106472755$. As we increase the Jacobi constant beyond this value, the minimum number of crossings that a homoclinic orbit can have with the section increases to 6 .

We want to argue that for any sufficiently large positive integer $N$, there exists a Lyapunov orbit whose stable and unstable manifolds do not intersect for the first $N$ revolutions around the mass, but they do intersect transversally after some $n>N$ revolutions (under the hypothesis that stable and the unstable manifolds of $L_{1}$ do not intersect, but they get arbitrarily close). Consider an integer $N$, the points $p_{1}, p_{2}, \ldots, p_{N}$ corresponding to the intersection number $1,2, \ldots, N$, respectively, of $W^{u}\left(L_{1}\right)$ with $\Pi^{+}$, and the points $p_{1}^{\prime}, p_{2}^{\prime}, \ldots, p_{N}^{\prime}$ corresponding to the intersection number $1,2, \ldots, N$, respectively, of $W^{s}\left(L_{1}\right)$ with $\Pi^{+}$. Choose pairwise disjoint disks $B_{p_{n}}(\epsilon), B_{p_{n}^{\prime}}(\epsilon)$ of radius $\epsilon>0, n=1, \ldots, N$, around these points, where $\epsilon$ is sufficiently small. Due to continuity with respect to initial conditions, there exists a Lyapunov orbit $T_{C}$ (very close to $L_{1}$ ), such that, for each $n=1,2, \ldots, N$, the $n$-th intersection between $W^{u}\left(T_{C}\right)$ and $\Pi^{+}$is contained in $B_{p_{n}}(\epsilon)$, and the $n$-th 
intersection between $W^{s}\left(T_{C}\right)$ and $\Pi^{+}$is contained in $B_{p_{n}^{\prime}}(\epsilon)$. These intersections are homeomorphic to a circle. Thus, there exists a Lyapunov orbit whose stable and unstable manifold crossings with the surface of section are all diffeomorphic copies of $S^{1}$ before the $(N+1)$-th crossing. Hence there is no homoclinic connection to $T_{C}$ before the $(N+1)$-th crossing. On the other hand, since we assume that the stable and unstable manifolds of $L_{1}$ fill densely a region on the Poincaré section, the manifolds of small Lyapunov orbits will intersect for a large enough number of cuts with the Poincaré section.

\section{SymboliC DYNAMiCS}

A discrete dynamical system $(X, f)$ is said to posses symbolic dynamics if there exits a finite or a countable set of symbols $S$, a homeomorphism $h: X \rightarrow S^{\mathbb{Z}}$, and a positive integer $k$ such $h \circ f^{k}=\sigma \circ h$. Here $S$ is provided with the discrete topology, the space of bi-infinite sequences $S^{\mathbb{Z}}$ is provided with the product topology, and the homeomorphism $\sigma: S^{\mathbb{Z}} \rightarrow S^{\mathbb{Z}}$ shifts every sequence one place to the left. The properties of the homeomorphism $h$ ensure that all orbits of $f$ can be matched with orbits of the shift map $\sigma$.

The existence of symbolic dynamics in the restricted three-body problem was proved analytically in several papers $[8,21,17,27]$ in the case when $\mu \approx 0$. The basic idea is to show analytically the existence of transverse homoclinic orbits to a periodic orbit at infinity. Then the Birkhoff-Smale homoclinic orbit theorem implies the existence of a horseshoe map, and, consequently, of symbolic dynamics. The existence of an invariant set of Cantor type for the horseshoe map implies that there cannot be any other analytic first integrals besides the Jacobi integral.

Our numerical investigations provide evidence for the existence of symbolic dynamics, and thus for the non-integrability of the problem in the case of $\mu=0.5$. For the energy level corresponding to $C=3.95, N=5$ is the smallest positive integer for which $\left(\Phi^{+}\right)^{N}$ is well defined on some subset of the surface of section. Let $q$ be one of the points $A, B, E, F, I, J$ corresponding to a homoclinic orbit with $N$ cuts (see Figure 12). By the Smale-Birkhoff homoclinic orbit theorem, there exists an $\left(\Phi^{+}\right)^{N}$-invariant set $\Lambda$ in a neighborhood of $q$ such that $\left(\Phi^{+}\right)^{N}$ restricted to $\Lambda$ is conjugate to a full shift on infinitely many symbols.

We can choose for example, a rectangle $R_{1}^{+}$by the point $B$, as in Figure 18 . The lower right hand side vertex corresponds to the homoclinic point $B$. One of the almost vertical sides of $R_{1}^{+}$is chosen very close to $W_{5}^{s}$, while one of the almost horizontal sides of $R_{1}^{+}$is chosen very close to $W_{1}^{u}$. Since $W_{1}^{u}$ is transverse to $W_{5}^{s}$, infinitely many components of $W_{6}^{u}$ intersect $W_{5}^{s}$ transversally. Thus, the image of $R_{1}^{+}$under $\Phi^{N}(N=5)$ crosses $R_{1}^{+}$in a horseshoe with infinitely many components, of the type shown in Figure 19. This shows the existence of symbolic dynamics over infinitely many symbols. There is a $\Phi^{N}$-invariant set $\Lambda_{1}^{+} \subset R_{1}^{+}$. Choosing the set of symbols $S=\mathbb{N}$, each point in $\Lambda_{1}^{+}$is associated to a unique sequence of symbols $\left(\ldots, n_{-2}, n_{-1}, n_{0}, n_{1}, n_{2}, \ldots\right)$. The terms $n_{0}, n_{1}, n_{2}, \ldots$ of the sequence describe an orbit that, in forward time, makes $n_{0}$ turns about the Lyapunov orbit followed by $N$ turns about the primary at $x=0.5$, then goes back to the Lyapunov orbit to make $n_{1}$ turns thereby, followed by another $N$ turns about the primary at $x=0.5$, then goes back again to the Lyapunov orbit to make $n_{2}$ turns thereby, followed by another $N$ turns around the primary at $x=0.5$, and so on. The terms $n_{-1}, n_{-2}, \ldots$ 


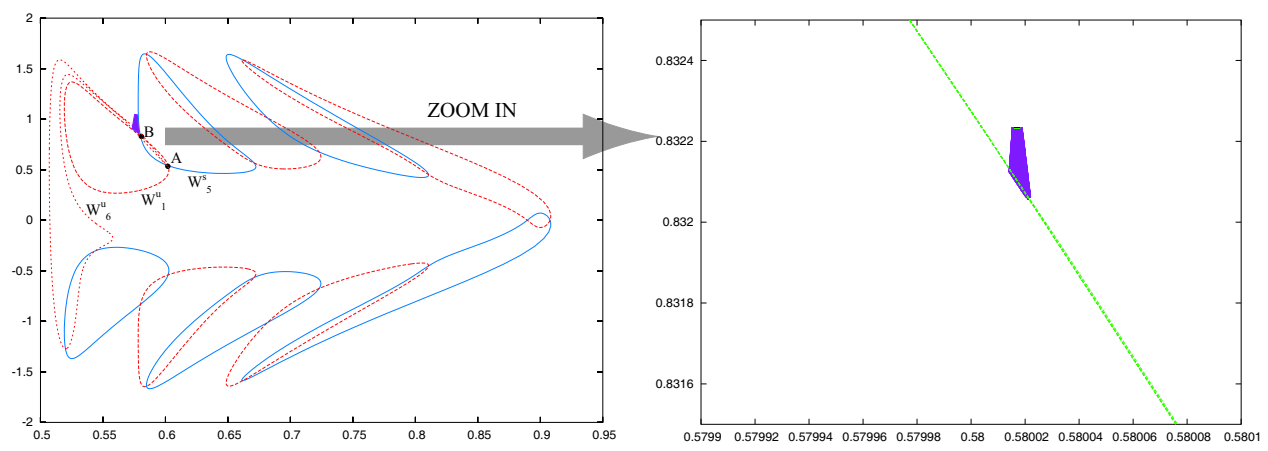

FIgURE 18. Rectangle mapped across itself by a horseshoe map (qualitative picture). The two open ended segments of $W_{6}^{u}$ converge to $W_{1}^{u}$ so fast that the branches of $\Phi^{5}\left(R_{1}\right)$ are almost indistinguishable.

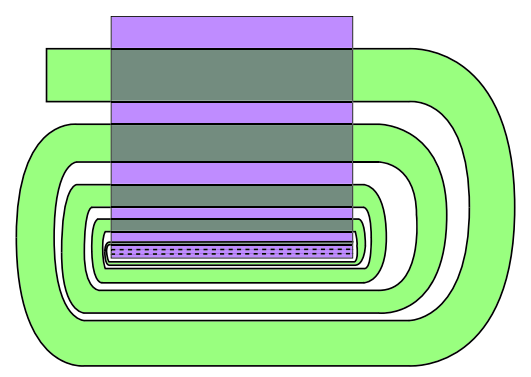

Figure 19. Infinite horseshoe (qualitative picture).
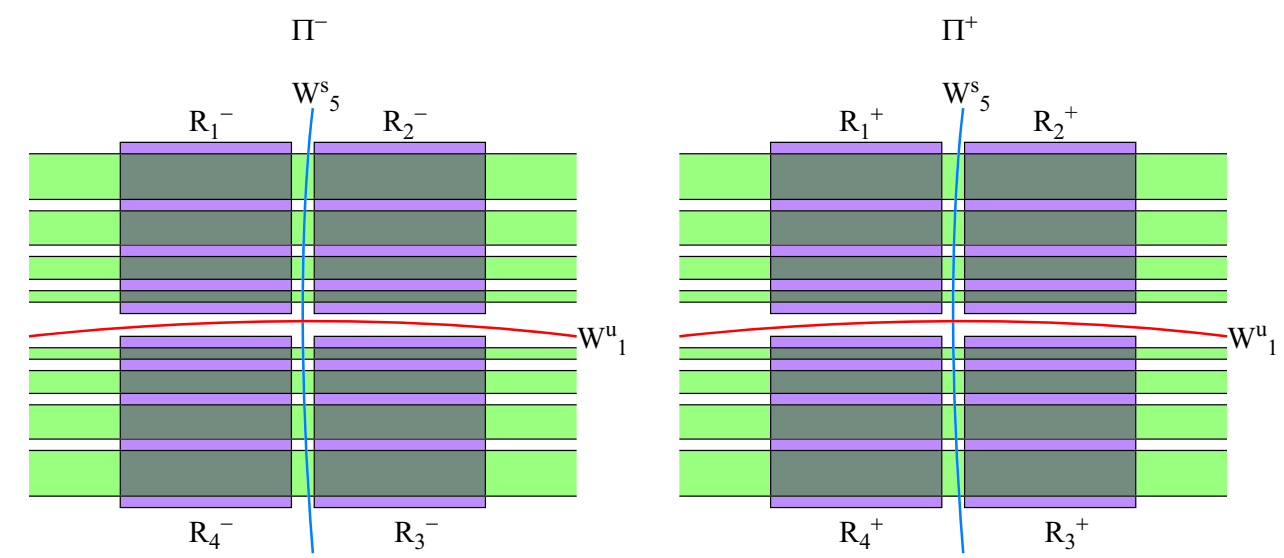

FiguRE 20. Rectangles and their images under $\Phi^{5}$ (qualitative picture).

describe the number of turns made about the Lyapunov orbit, intermingled with sequences of $N$ turns about the primary at $x=0.5$, done in the past. 
The rectangle $R_{1}^{+}$was chosen outside the 'circles' $W_{1}^{u}$ and $W_{5}^{s}$. We can construct similar rectangles: $R_{2}^{+}$outside $W_{1}^{u}$ and inside $W_{5}^{s}, R_{3}^{+}$inside both $W_{1}^{u}$ and $W_{5}^{s}$, and $R_{4}^{+}$inside $W_{1}^{u}$ and outside $W_{5}^{s}$. We can also construct the counterparts of these rectangles $R_{1}^{-}, R_{2}^{-}, R_{3}^{-}, R_{4}^{-}$in the Poincaré section $\Pi^{-}$in $x<-0.5$. They are illustrated schematically in Figure 20. We can associate symbolic dynamics to all orbits that visit the rectangles $R_{1}^{+}, R_{2}^{+}, R_{3}^{+}, R_{4}^{+}, R_{1}^{-}, R_{2}^{-}, R_{3}^{-}, R_{4}^{-}$. This symbolic dynamics describes the typical 'acrobatics' that an orbit can do.

The past and the future orbits of points in $R_{1}^{+}, R_{2}^{+}, R_{3}^{+}, R_{4}^{+}, R_{1}^{-}, R_{2}^{-}, R_{3}^{-}, R_{4}^{-}$is dictated by the fact that the stable and unstable manifolds are separatrices of the phase space in the sense described in Section 5. The behavior of such points can be summarized by the following tables:

\begin{tabular}{|c|c|c|}
\hline & comes from & $\begin{array}{c}\text { after } N \text { turns } \\
\text { around } x=-0.5 \\
\text { goes to }\end{array}$ \\
\hline$R_{1}^{-}$ & $x<0$ & $x<0$ \\
$R_{2}^{-}$ & $x<0$ & $x>0$ \\
$R_{3}^{-}$ & $x>0$ & $x>0$ \\
$R_{4}^{-}$ & $x>0$ & $x<0$ \\
\hline
\end{tabular}

\begin{tabular}{|c|c|c|}
\hline & comes from & $\begin{array}{c}\text { after } N \text { turns } \\
\text { around } x=0.5 \\
\text { goes to }\end{array}$ \\
\hline$R_{1}^{+}$ & $x>0$ & $x>0$ \\
$R_{2}^{+}$ & $x>0$ & $x<0$ \\
$R_{3}^{+}$ & $x<0$ & $x<0$ \\
$R_{4}^{+}$ & $x<0$ & $x>0$ \\
\hline
\end{tabular}

The possible transitions can be represented by the following directed graph:

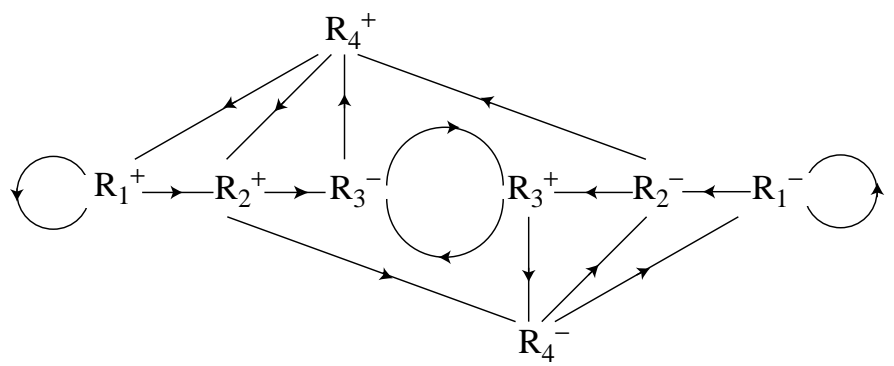

Each arrow in the graph represents a visit to the Lyapunov orbit that makes some arbitrary number $n_{i}$ of turns over there, where $i>0$ refers to the future trajectory and $i<0$ refers to the past trajectory of a point originating in one of the rectangles. When the arrow joins $R_{j}^{+}$to $R_{k}^{+}$the arrow represents $\Phi^{N}$; this is a map that takes a point $x \in \Pi^{+} \cup \Pi^{-}$, performs $N$ revolutions about the primary in the region of departure, and lands again at $\Phi^{n}(x) \in \Pi^{+} \cup \Pi^{-}$. When the point is taken from $\Pi^{+}$and lands in $\Pi^{+}$, the map is $\Phi^{N}=\left(\Phi^{+}\right)^{N}$; when the point is taken from $\Pi^{-}$and lands in $\Pi^{-}$, the map is $\Phi^{N}=\left(\Phi^{-}\right)^{N}$.

$\left(\Phi^{+}\right)^{N}$, when it joins $R_{j}^{-}$to $R_{k}^{-}$the arrow represents $\left(\Phi^{-}\right)^{N}$, when it joins $R_{j}^{+}$ to $R_{k}^{-}$or when it joins $R_{j}^{-}$to $R_{k}^{+}$the arrow represents the arrow represents $\Phi^{N}$.

Similar symbolic dynamics is carefully described and argued in $[2,16]$.

\section{BeHAVIOR OF THE HOMOCLINIC ORBITS TO LyAPUNOV ORBITS AS THE ENERGY LEVEL APPROACHES THAT OF $L_{1}$}

Homoclinic orbits can be classified in terms of the number of turns they make about a primary. This section is concerned with homoclinic orbits that remain in $x>0$ and make the smallest number of turns about a primary at a given energy 
level. Homoclinic orbits with lowest number of turns around the primary are of interest in astrodynamics since they provide zero cost trajectories which approach asymptotically a Lyapunov orbit in both forward and backwards time, while they minimize the time they spend around the libration point and around a primary. See $[5]$.

We perform numerical explorations for Jacobi constants $C_{2}<C<C_{1}$, corresponding to Hill's regions that have the dynamical channels at $L_{2}$ and $L_{3}$ closed, and the dynamical channel at $L_{1}$ open. For a wide range of energy levels corresponding to $C_{2}<C<C_{1}$, there exist infinitely many homoclinic orbits, making various numbers of turns. From all homoclinic orbits, we will search for the 'shortest' ones, i.e., those which make the smallest number of turns about the primary at $x=1 / 2$. For each $C$ in the range, we generate $W_{i}^{s}$ and $W_{j}^{u}$ up to some reasonably large number of cuts and then look for the intersections that give the minimal value of $i+j-1$. When $C$ approaches $C_{1}$ and the Lyapunov orbits get smaller and smaller, the cuts made by the stable and unstable manifolds off the surface of section resemble more and more to the 'fish' (see Figure 7). The whole range of Jacobi constants $C_{2}<C<C_{1}$ can be naturally partitioned into intervals such that, for each $C$ in an interval, the number of turns made by the shortest homoclinic orbits remains constant. We will describe mechanisms that produce homoclinic orbits with the smallest number of turns for $C_{2}<C<C_{1}$. The geometry of these mechanisms will be described in relation to the anatomy of the 'fish'. Here we make an amusing remark that the 'fish' is anatomically correct (see Figure 25). We will not attempt to find each range of Jacobi constants for which this number of turns stays constant.

We first describe the behavior of homoclinic orbits for Jacobi constants $C$ slightly larger than $C_{2}$; we start with the value $C_{2}+\varepsilon$, where we choose $\varepsilon=10^{-6}$. For $C_{2}+\varepsilon<C<3.90$, the stable and unstable manifolds of the Lyapunov orbits exhibit collisions and close encounters with the mass at $\mu$. Therefore KS (LeviCivita) regularization has been used to avoid ill conditioning and increase of errors during numerical integration (see [25] for the classical theory).

For $C<3.64309$, the stable manifold $W^{s}\left(T_{C}\right)$ and the unstable manifold $W^{u}\left(T_{C}\right)$ collide with the mass $\mu$ at the first encounter with $y=0, x<0.5$ or with $y=0, x>$ 0.5. For $C>3.64208$, the stable and unstable manifold intersect the first time they meet $\Pi^{+}$, producing homoclinic orbits that make 1 turn about the mass. See Figure 21.

As $C$ increases, the invariant manifolds cease to collide with the mass while they still intersect along homoclinic orbits making 1 turn about the mass. At $C \approx 3.845065165, W_{1}^{s}$ and $W_{1}^{u}$ cease to intersect, and the shortest homoclinic orbits make 2 turns around the mass. They occur as intersection of $W_{2}^{s}$ and $W_{1}^{u}$, and as intersection of $W_{1}^{s}$ and $W_{2}^{u}$. This trend continues as $C$ increases from 3.64208 to 3.90 , and the numbers of turns made by the shortest homoclinic orbits is given by the sequence

\section{$1,2,3,4$.}

The corresponding orbits are realized as intersections between $W_{1}^{s}$ and $W_{1}^{u}, W_{1}^{s}$ and $W_{2}^{u}$ (and also $W_{2}^{s}$ and $W_{1}^{u}$ ), $W_{2}^{s}$ and $W_{2}^{u}$, and $W_{2}^{s}$ and $W_{3}^{u}$ (and also $W_{3}^{s}$ and $\left.W_{2}^{u}\right)$, respectively.

When $C>3.90$, the stable and unstable manifolds can be integrated without using regularization. The shortest homoclinic orbits are obtained as the intersection 


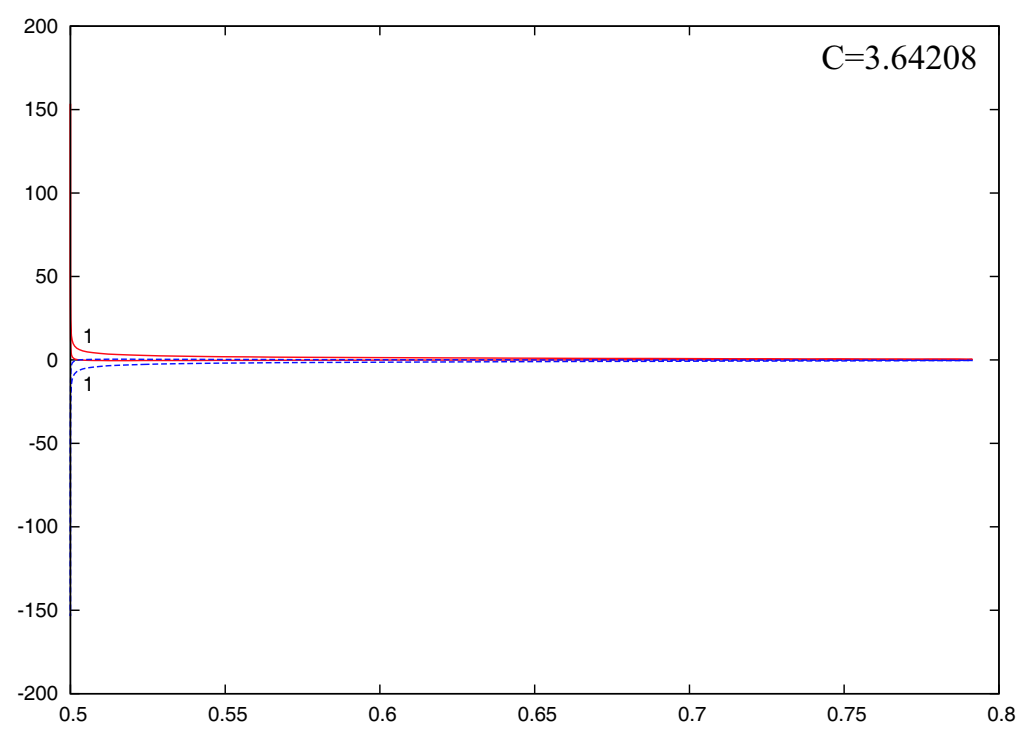

Figure 21. Stable and unstable manifolds colliding with the primary.

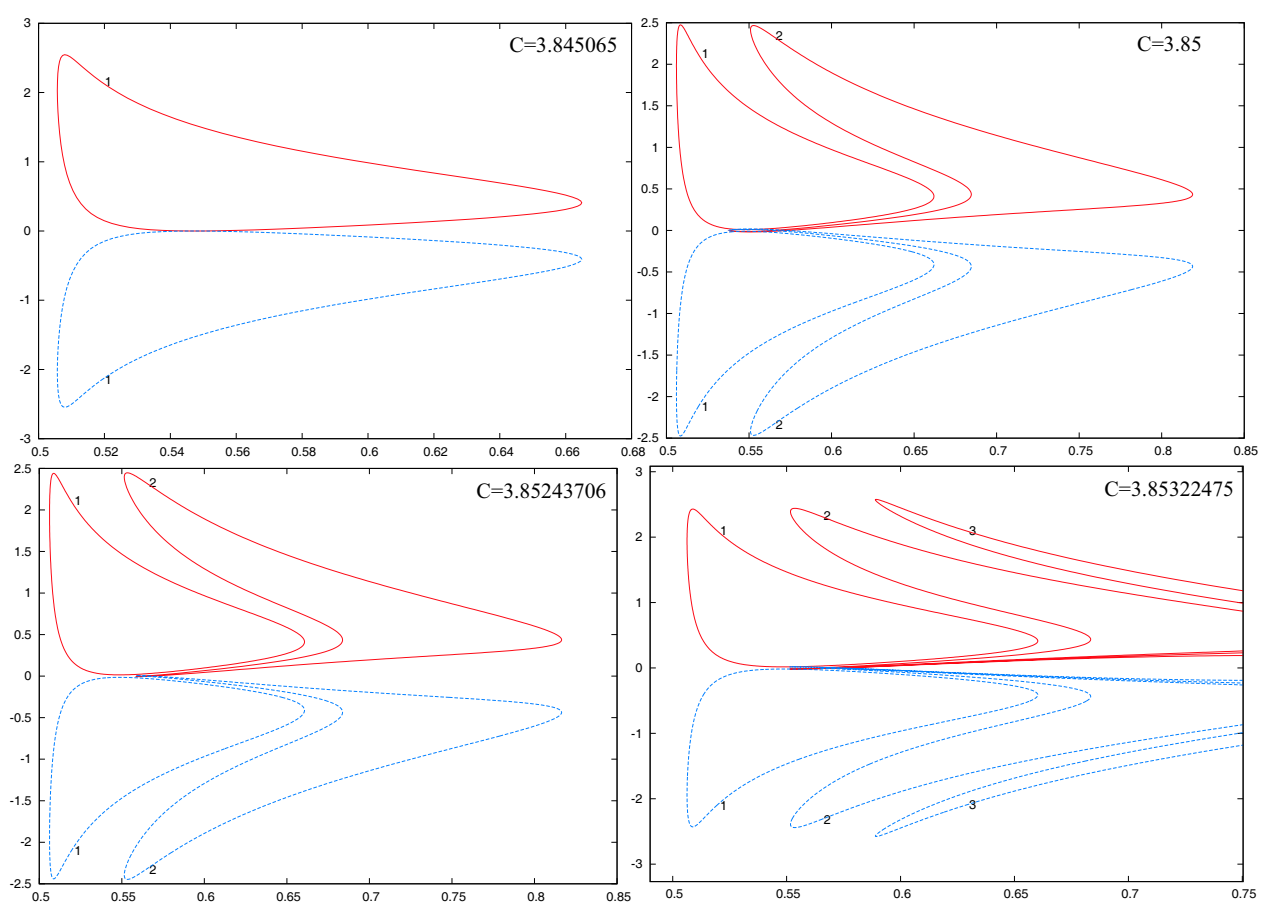

Figure 22. Poincaré section of homoclinic orbits making $1,2,3,4$ turns about the primary. 

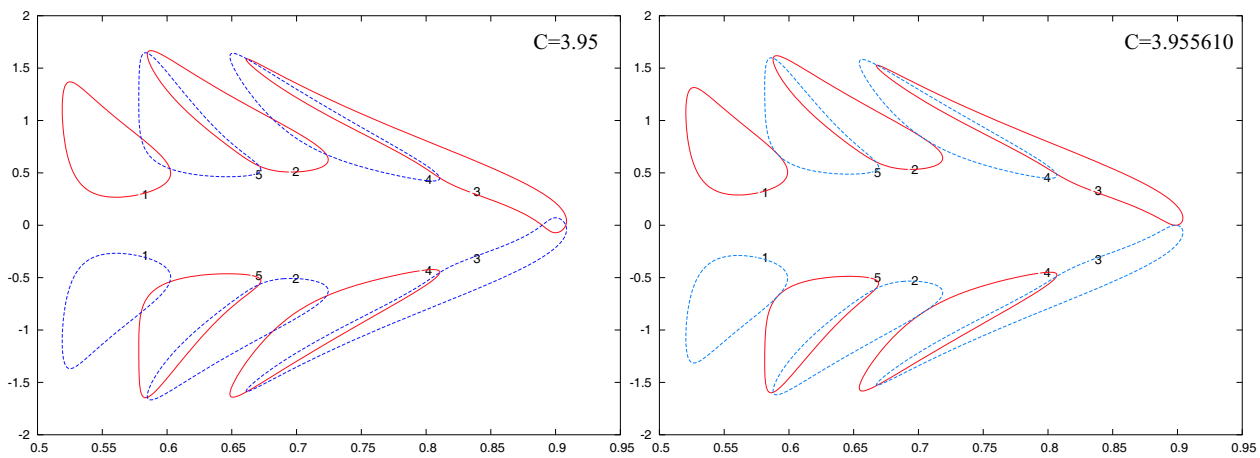

FIGURE 23. Poincaré section of homoclinic orbits with the smallest number of turns equal to 5 .

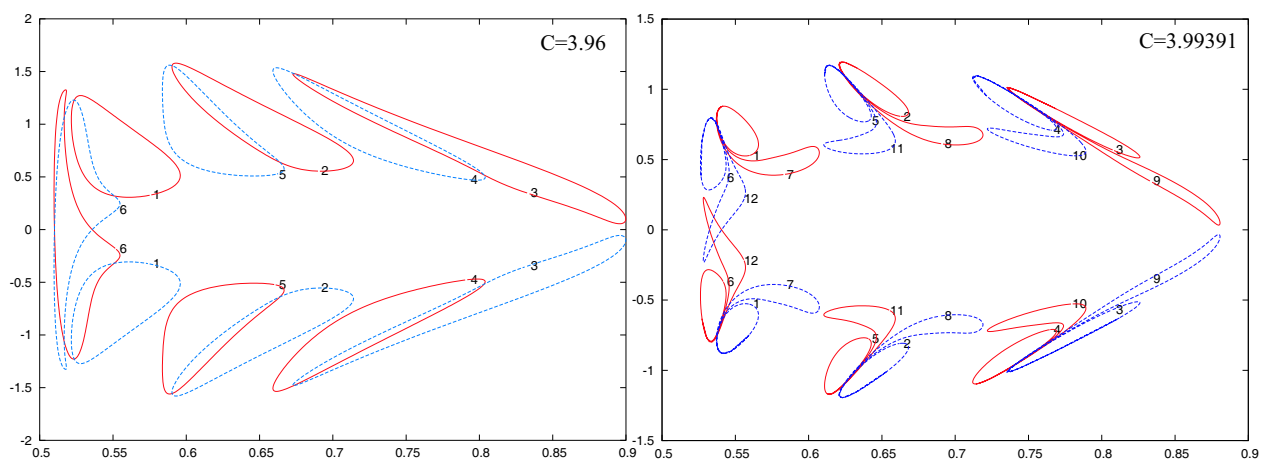

FiguRE 24. Poincaré section of homoclinic orbits with the smallest number of turns equal to 6 . Intersections between $W_{6}^{s}$ and $W_{1}^{u}$ are observed. On the right, $W_{6}^{s}$ and $W_{1}^{u}$ are tangent.

of $W_{1}^{u}$ with $W_{5}^{s}$, and make 5 turns bout the primary. For $3.90<C<3.955610$, the smallest number of turns that a homoclinic orbit can have remains 5 . At $C \approx 3.955610$, the cuts $W_{1}^{u}$ and $W_{5}^{s}$ meet tangentially. See Figure 23. When $C$ is increased beyond 3.955610, the cuts $W_{1}^{u}$ and $W_{5}^{s}$ cease to meet. The smallest number of turns that a homoclinic orbit can have increases to 6 . See Figure 24 . Homoclinic orbits with 6 cuts are obtained as intersections of $W_{1}^{u}$ with $W_{6}^{s}$.

We now describe a mechanism that produces homoclinic orbits for the range $3.995610<C<3.999690$. There are two types of orbits produced. The first type are orbits that occur by the middle of the 'tail-fin of the fish' (see Figure 25), as possible intersections of $W_{6 n}^{s}$ and $W_{6 n+6}^{s}$ with $W_{6 n}^{u}$ and $W_{6 n+6}^{u}$, for $n \geq 1$. These possible intersections result in homoclinic orbits that make $12 n-1,12 n+5$ and $12 n+11$ turns about the primary. The orbits that make $12 n-1$ or $12 n+11$ turns are symmetric orbits (with respect to $(x, y, \dot{x}, \dot{y}) \rightarrow(x,-y,-\dot{x}, \dot{y}))$, and those that make $12 n+5$ turns are non-symmetric orbits. The second type are orbits that occur by the upper half of the 'tail-fin of the fish' (see Figure 25), as possible intersections of $W_{6 m}^{s}$ and $W_{6 m+6}^{s}$ with $W_{6 m+1}^{u}$ and $W_{6 m+7}^{u}$, for $m \geq 1$. These possible intersections result in homoclinic orbits that make $12 m, 12 m+6$ and $12 m+12$ turns about the primary. They are all non-symmetric orbits. All homoclinic orbits described 


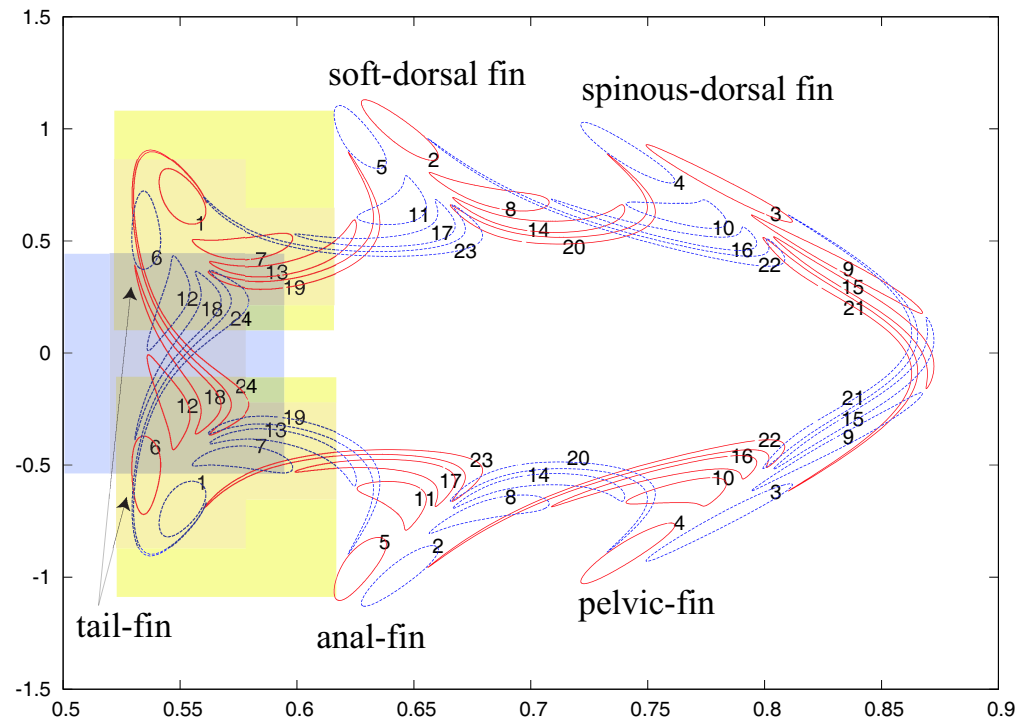

FiguRE 25. Poincaré section of homoclinic orbits formed in the middle of the 'tail-fin' and the upper/lower half of the 'tail-fin' of the 'fish'. Intersections between $W_{18}^{s}$ and $W_{18}^{u}, W_{24}^{s}$ and $W_{24}^{u}$, $W_{18}^{s}$ and $W_{24}^{u}, W_{24}^{s}$ and $W_{19}^{u}$, and of their symmetric counterparts, are observed. They correspond to homoclinic orbits that make $29=12 \cdot 2+5,35=12 \cdot 2+11,42=12 \cdot 3+6$ turns about the primary. Intersections between $W_{6}^{s}$ and $W_{6}^{u}$, and $W_{12}^{s}$ and $W_{12}^{u}$, for example, cease to exist at this energy level.

above have counterparts in other regions of the 'fish': in the 'soft dorsal fin', in the 'spinous dorsal fin', in the 'pelvic fin', in the 'anal fin', and in the lower half of the 'tail-fin of the fish'.

The shortest homoclinic orbits obtained through this mechanism are either of the first type or of the second type.

In the first stage, both types of orbits described above compete for the shortest homoclinic orbit. Consider, for example, $C=3.99391$. There exist intersections of $W_{12}^{s}$ with $W_{12}^{u}$. This intersections result in a pair of symmetric homoclinic orbits that make 23 turns about the primary. There also exist intersections of $W_{6}^{s}$ with $W_{1}^{u}$ and $W_{7}^{u}$, and of $W_{12}^{s}$ with $W_{1}^{u}$ and $W_{7}^{u}$. These intersections result in pairs of homoclinic orbits that make 6,12 and 18 turns about the primary. The homoclinic orbits with the smallest number of turns are those making 6 turns.

As $C$ is increased, at some point $W_{6}^{s}$ and $W_{1}^{u}$ cease to intersect. See Figure 26. The corresponding sequence of the smallest number of turns made by a homoclinic orbit evolves as

$$
12,18 .
$$

In the second stage, starting with $C=3.99391$, the homoclinic orbits with the smallest number of turns only occur in the middle of the 'tail-fin of the fish'. They are orbits resulting from the possible intersections of $W_{6 n}^{s}$ and $W_{6 n+6}^{s}$ with $W_{6 n}^{u}$ and $W_{6 n+6}^{u}, n \geq 2$. There are two possible cases. One case is when $W_{6 n}^{s}$ intersects with $W_{6 n}^{u}, W_{6 n}^{s}$ intersects with $W_{6 n+6}^{u}, W_{6 n+6}^{s}$ intersects with $W_{6 n}^{u}$, and $W_{6 n+6}^{s}$ 


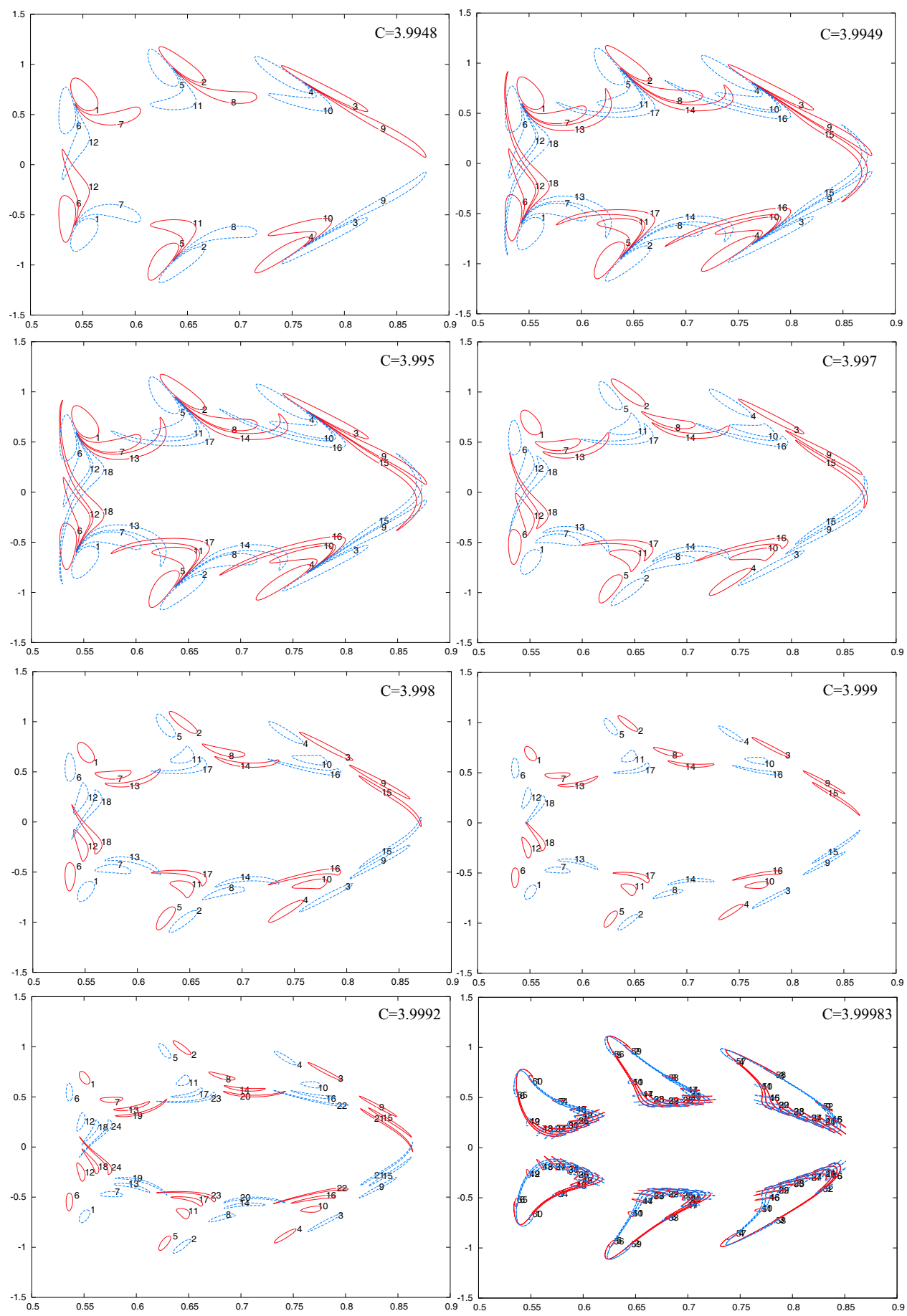

Figure 26. Poincaré section of homoclinic orbits with the smallest number of turns at least 12 . 

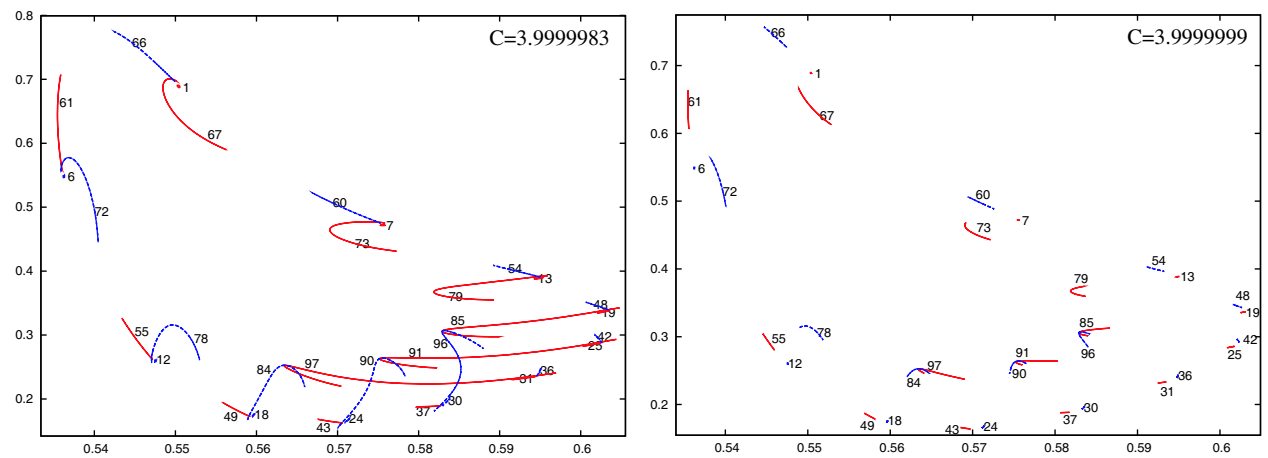

FIGURE 27. New mechanisms competing in generating homoclinic orbits with the smallest number of turns.

intersects with $W_{6 n+6}^{u}$. Then the homoclinic orbits with the smallest numbers of turns about the primary are those with $12 n-1$ turns. The other case is when $W_{6 n}^{s}$ does not intersect with $W_{6 n}^{u}, W_{6 n}^{s}$ intersects with $W_{6 n+6}^{u}, W_{6 n+6}^{s}$ does not intersect with $W_{6 n}^{u}$, and $W_{6 n+6}^{s}$ intersects with $W_{6 n+6}^{u}$. Then the homoclinic orbits with the smallest numbers of turns about the primary are those with $12 n+5$ turns.

Consider, for example, $C=3.995$. There exist intersections of $W_{12}^{s}$ with $W_{12}^{u}$, of $W_{12}^{s}$ with $W_{18}^{u}$, of $W_{18}^{s}$ with $W_{12}^{u}$, and of $W_{18}^{s}$ with $W_{18}^{u}$. These intersections result in pairs homoclinic orbits that make 23,29 and 35 turns about the primary. There also exist intersections of $W_{12}^{s}$ with $W_{19}^{u}$, of $W_{18}^{s}$ with $W_{13}^{u}$, and of $W_{18}^{s}$ with $W_{19}^{u}$. These intersections result in pairs of homoclinic orbits that make 30 and 36 turns about the primary. The homoclinic orbits with the smallest number of turns about the primary are those with 23 turns. Now, consider $C=3.997$. There exist intersections of $W_{12}^{s}$ with $W_{18}^{u}$, of $W_{18}^{s}$ with $W_{12}^{u}$, and of $W_{18}^{s}$ with $W_{18}^{u}$. These intersections result in pairs of homoclinic orbits that make 29 and 35 turns about the primary. There also exist intersections of $W_{18}^{s}$ with $W_{25}^{u}$, of $W_{24}^{s}$ with $W_{19}^{u}$, and of $W_{24}^{s}$ with $W_{25}^{u}$. These intersections result in pairs homoclinic orbits that make 42,48 and 52 turns about the primary. The homoclinic orbits with the smallest number of turns about the primary are those with 29 turns.

The corresponding sequence of the smallest number of turns made by a homoclinic orbits is

$$
23,29,35,41,47,53 \text {. }
$$

In the third stage, the shortest homoclinic occur only in the upper half of the 'tail-fin of the fish'. The corresponding sequence of the smallest number of turns made by a homoclinic orbits continues as

$$
54,60,66 \text {. }
$$

In conclusion, the sequence of the smallest numbers of turns made by homoclinic orbits about the primary that we found so far is

$$
1,2,3,4,5,6,12,18,23,29,35,41,47,53,54,60,66 \text {. }
$$

When $C$ is increased further, it appears that new mechanisms may compete for producing the shortest homoclinic orbits. The geometry of the cuts made by the stable and unstable manifolds with the Poincaré section in the upper half of the 'tail-fin of the fish' indicates two rows of cuts where the competition for the 
shortest homoclinic orbits seems to have moved. For some values of $C$, the shortest homoclinic orbits are among the intersections between cuts in the outward row and cuts in the inward row. This is the case, for example, when $C=3.9999983$, when the shortest homoclinic orbits makes 132 turns. Increasing $C$ further, the shortest homoclinic orbits occur exclusively as intersections among cuts in the inward row. This is the case, for example, when $C=3.9999999$, when the shortest homoclinic orbits makes 180 turns. See Figure 27.

Of course, as we keep increasing $C$, some aspects of the numerical computations need a careful check. We have decided to stop them at $C=C_{1}-\epsilon$ where $\epsilon=$ $10^{-6}$ because, although we could proceed somewhat further, the lack of theoretical support about the behavior of the manifolds of $L_{1}$ will constitute at some point an obstruction to the resolution of our computations, independently of the numerical procedure used.

\section{ACKNOWLEDGEMENT}

Part of this work was done during visits made by M.G. to Departament de Matemàtica Aplicada I, Universitat Politècnica de Catalunya, for whom hospitality he is very grateful. M.G. would also like to thank Richard Moeckel for useful discussions.

\section{REFERENCES}

[1] R. Abraham and J. Marsden, Foundations of Mechanics, Addison Wesley, Reading, 1985.

[2] D.F. Appleyard, Invariant Sets near the Collinear Lagrangian Points of the Nonplanar Restricted Three Body Problem, PhD Thesis, University of Wisconsin, 1970.

[3] E. Belbruno, Capture dynamics and chaotic motions in celestial mechanics: With applications to the construction of low energy transfers, Princeton University Press, Princeton, NJ, 2004.

[4] J.M. Blondin, M.T. Richards and M.L. Malinowski, Hydrodynamic simulations of the mass transfer in Algol, Astrophys. J. 445 (1995), pp. 939-946.

[5] E. Canalias and J.J. Masdemont, Homoclinic and Heteroclinic Transfer Trajectories Between Planar Lyapunov Orbits in the Sun-Earth and Earth-Moon Systems, Discrete and Continuous Dynamical Systems 14 (2006), pp. 261-279.

[6] C.C. Conley, Low energy transit orbits in the restricted three-body problem, SIAM J. Appl. Math. 16 (1968), pp. 732-746.

[7] C.C. Conley, Isolated Invariant Sets and the Morse Index, CBMS Regional Conference Series 38, American Mathematical Society, 1978.

[8] R.W. EASTON AND R. MCGeheE, Homoclinic phenomena for orbits doubly asymptotic to an invariant three-sphere, Indiana Univ. Math. J. 28 (1979), pp. 211-240.

[9] M. Dellnitz, O. Junge, M.W. Lo, J.E. Marsden, K. Padberg, R. Preis, S.D. Ross, B. ThIERE, Transport of Mars-Crossers from the Quasi-Hilda Region, Physical Review Letters 94 (2005), pp. (231102)1-4.

[10] M. Dellnitz, O. Junge, W.S. Koon, F. Lekien, M.W. Lo, J.E. Marsden, K. Padberg, R. Preis, S.D. Ross, B. Thiere, Transport in Dynamical Astronomy and Multibody Problems, International Journal of Bifurcation and Chaos, International Journal of Bifurcation and Chaos 15 (2005), pp. 699-727.

[11] M. Gidea And M. Burgos, Chaotic transfers in three- and four-body systems, Physica A 328 (2003), pp. 360-366.

[12] G. Gómez, J. Llibre, J.J. Masdemont, Homoclinic and Heteroclinic Solutions in the Restricted Three-Body Problem, Celestial Mechanics 44 (1988), pp. 239-259.

[13] G. Gómez, J. Masdemont, and J.M. Mondelo, Libration Point Orbits: A Survey from the Dynamical Point of View, in Libration Point Orbits and Applications, Proceedings 
of the Conference Aiguablava, Spain, 10 - 14 June 2002, ed. G. Gómez, M.W. Lo, and J.J. Masdemont, World Scientific, 2003.

[14] M.J. Holman and P.A. Wiegert, Long-Term Stability of Planets in Binary Systems, Astron. J. 117 (1999), pp. 621-628. Astron. J., 117 (1999), 621.

[15] A. Jorba, J.J. Masdemont, Dynamics in the Center Manifold of the Collinear Points of the Restricted Three Body Problem, Physica D 132 (1999), pp. 189-213.

[16] W.S. Koon, M.W. Lo, J.E. Marsden, and S.D. Ross, Heteroclinic connections between periodic orbits and resonance transitions in celestial mechanics, Chaos, 10 (2000), pp. 427469.

[17] J. Llibre And C. Simó, Oscillatory solutions in the planar restricted three-body problem, Math. Ann. 248 (1980), pp. 153-184.

[18] S. H. Lubow and F. H. Shu, Gas Dynamics of Semidetached Binaries, The Astrophysical Journal 198 (1975), pp. 383-405.

[19] J. Llibre , R. MarTínez AND C. Simó, Tranversality of the invariant manifolds associated to the Lyapunov family of periodic orbits near $L_{2}$ in the restricted three-body problem, J. Differential Equations 58 (1985), 104-156.

[20] J.J. Masdemont, High Order Expansions of Invariant Manifolds of Libration Point Orbits with Applications to Mission Design associated with Collinear Lissajous Libration Orbits, Dynamical Systems an International Journal, to appear.

[21] J. Moser, Stable and Random Motions in Dynamical Systems, Princeton University Press, Princeton, NJ, 1973.

[22] C. OcAmpo, An Architecture for a Generalized Spacecraft Trajectory Design and Optimization System, in Libration Point Orbits and Applications, Proceedings of the Conference Aiguablava, Spain, 10 - 14 June 2002, ed. G. Gómez, M.W. Lo, and J.J. Masdemont, World Scientific, 2003.

[23] D.L. Richardson, A note on the Lagrangian Formulation for Motion about Collinear Points, Celestial Mechanics, 22 (1980), pp. 231-235.

[24] C. Simó And T.J. Stuchi, Central stable/unstable manifolds and the destruction of KAM tori in the planar Hill problem, Phys. D, 140 (2000), pp. 1-32.

[25] E. L. Stiefel, G. Scheifele, Linear and Regular Celestial Mechanics, SpringerVerlag, Berlin, Heidelberg, NY, 1971.

[26] V. Szebehely, Theory of Orbits, Academic Press, Orlando, 1967.

[27] Z. XIA, Melnikov Methods and Transversal Homoclinic Points in the Restricted Three-Body Problem, J. Diff. Eqns., 96 (1992), pp. 170-184.

Department of Mathematics, Northeastern Illinois University, Chicago, IL 60625, U.S.A.

E-mail address: mgidea@neiu.edu

Departament de Matemàtica Aplicada i, Universitat Politècnica de Catalunya,

E.T.S.E.I.B, Diagonal 647, 08028 Barcelona, Spain

E-mail address: josep@barquins.upc.edu 